\title{
DETECTION OF THE BARYON ACOUSTIC PEAK IN THE LARGE-SCALE CORRELATION FUNCTION OF SDSS LUMINOUS RED GALAXIES
}

\author{
Daniel J. Eisenstein, ${ }^{1,2}$ Idit Zehavi, ${ }^{1}$ David W. Hogg, ${ }^{3}$ Roman Scoccimarro, ${ }^{3}$ Michael R. Blanton, ${ }^{3}$ Robert C. Nichol, \\ Ryan Scranton, ${ }^{5}$ Hee-Jong Seo, ${ }^{1}$ Max Tegmark, ${ }^{6,7}$ Zheng Zheng, ${ }^{8}$ Scott F. Anderson, ${ }^{9}$ Jim Annis, ${ }^{10}$ Neta Bahcall, ${ }^{11}$ \\ Jon Brinkmann, ${ }^{12}$ Scott Burles, ${ }^{7}$ Francisco J. Castander, ${ }^{13}$ Andrew Connolly, ${ }^{5}$ Istvan Csabai, ${ }^{14}$ Mamoru Doi, ${ }^{15}$ \\ Masataka Fukugita, ${ }^{16}$ Joshua A. Frieman, ${ }^{10,17}$ Karl Glazebrook, ${ }^{18} \mathrm{James}_{\text {E. Gunn, }}{ }^{11}$ John S. Hendry, ${ }^{10}$ \\ Gregory Hennessy, ${ }^{19}$ Zeljko Ivezić, ${ }^{9}$ Stephen Kent, ${ }^{10}$ Gillian R. Knapp, ${ }^{11}$ Huan Lin, ${ }^{10}$ Yeong-Shang Loh, ${ }^{20}$ \\ Robert H. Lupton, ${ }^{11}$ Bruce Margon, ${ }^{21}$ Timothy A. McKay, ${ }^{22}$ Avery Meiksin, ${ }^{23}$ Jeffery A. Munn, ${ }^{19}$ \\ Adrian Pope, ${ }^{18}$ Michael W. Richmond, ${ }^{24}$ David Schlegel, ${ }^{25}$ Donald P. Schneider, ${ }^{26}$ \\ Kazuhiro Shimasaku, ${ }^{27}$ Christopher Stoughton, ${ }^{10}$ Michael A. Strauss, ${ }^{11}$ \\ Mark SubbaRao, ${ }^{17,28}$ Alexander S. Szalay, ${ }^{18}$ István Szapudi, ${ }^{29}$ \\ Douglas L. Tucker, ${ }^{10}$ Brian Yanny, ${ }^{10}$ and Donald G. York ${ }^{17}$ \\ Received 2004 December 31; accepted 2005 July 15
}

\begin{abstract}
We present the large-scale correlation function measured from a spectroscopic sample of 46,748 luminous

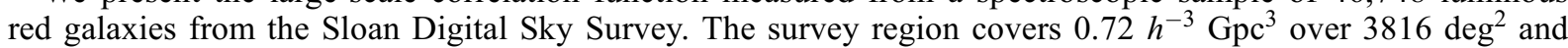
$0.16<z<0.47$, making it the best sample yet for the study of large-scale structure. We find a well-detected peak in the correlation function at $100 \mathrm{~h}^{-1} \mathrm{Mpc}$ separation that is an excellent match to the predicted shape and location of the imprint of the recombination-epoch acoustic oscillations on the low-redshift clustering of matter. This detection demonstrates the linear growth of structure by gravitational instability between $z \approx 1000$ and the present and confirms a firm prediction of the standard cosmological theory. The acoustic peak provides a standard ruler by which we can measure the ratio of the distances to $z=0.35$ and $z=1089$ to $4 \%$ fractional accuracy and the absolute distance to $z=0.35$ to $5 \%$ accuracy. From the overall shape of the correlation function, we measure the matter density $\Omega_{m} h^{2}$ to $8 \%$ and find agreement with the value from cosmic microwave background (CMB) anisotropies. Independent of the constraints provided by the CMB acoustic scale, we find $\Omega_{m}=0.273 \pm 0.025+0.123\left(1+w_{0}\right)+$ $0.137 \Omega_{K}$. Including the CMB acoustic scale, we find that the spatial curvature is $\Omega_{K}=-0.010 \pm 0.009$ if the dark energy is a cosmological constant. More generally, our results provide a measurement of cosmological distance, and hence an argument for dark energy, based on a geometric method with the same simple physics as the microwave background anisotropies. The standard cosmological model convincingly passes these new and robust tests of its fundamental properties.
\end{abstract}

Subject headings: cosmic microwave background — cosmological parameters - cosmology: observations — distance scale - galaxies: elliptical and lenticular, $\mathrm{cD}$ - large-scale structure of universe

Online material: color figures

\footnotetext{
1 Steward Observatory, University of Arizona, 933 North Cherry Avenue, Tucson, AZ 85121.

2 Alfred P. Sloan Fellow.

3 Center for Cosmology and Particle Physics, Department of Physics, New York University, 4 Washington Place, New York, NY 10003.

4 Institute of Cosmology and Gravitation, Mercantile House, Hampshire Terrace, University of Portsmouth, Portsmouth P01 2EG, UK.

5 Department of Physics and Astronomy, University of Pittsburgh, Pittsburgh, PA 15260.

6 Department of Physics, University of Pennsylvania, Philadelphia, PA 19104.

7 Department of Physics, Massachusetts Institute of Technology, Cambridge, MA 02139.

8 School of Natural Sciences, Institute for Advanced Study, Princeton, NJ 08540.

9 Department of Astronomy, University of Washington, Box 351580, Seattle, WA 98195-1580.

${ }^{10}$ Fermilab National Accelerator Laboratory, P.O. Box 500, Batavia, IL 60510.

11 Princeton University Observatory, Peyton Hall, Princeton, NJ 08544.

12 Apache Point Observatory, P.O. Box 59, Sunspot, NM 88349.

13 Institut d'Estudis Espacials de Catalunya/CSIC, Gran Capità 2-4, E-08034 Barcelona, Spain.

14 Department of Physics of Complex Systems, Eötvös University, Pázmány Péter sétány 1, H-1518 Budapest, Hungary.

15 Institute of Astronomy, School of Science, University of Tokyo, 2-21-1 Osawa, Mitaka, Tokyo 181-0015, Japan.

16 Institute for Cosmic Ray Research, University of Tokyo, Kashiwa 277-8582, Japan.

17 University of Chicago, Astronomy and Astrophysics Center, 5640 South Ellis Avenue, Chicago, IL 60637.

18 Department of Physics and Astronomy, Johns Hopkins University, 3701 San Martin Drive, Baltimore, MD 21218.

19 United States Naval Observatory, Flagstaff Station, P.O. Box 1149, Flagstaff, AZ 86002.

20 Center for Astrophysics and Space Astronomy, University of Colorado, Boulder, CO 80803.

21 Space Telescope Science Institute, 3700 San Martin Drive, Baltimore, MD 21218.

22 Department of Physics, University of Michigan, Ann Arbor, MI 48109-1120.

23 Institute for Astronomy, University of Edinburgh, Royal Observatory, Blackford Hill, Edinburgh EH9 3HJ, UK.

24 Department of Physics, Rochester Institute of Technology, 85 Lomb Memorial Drive, Rochester, NY $14623-5603$.

25 Lawrence Berkeley National Laboratory, 1 Cyclotron Road, MS 50R-5032, Berkeley, CA 94720-8160.

26 Department of Astronomy and Astrophysics, Pennsylvania State University, University Park, PA 16802.

27 Department of Astronomy, School of Science, University of Tokyo, 7-3-1 Hongo, Bunkyo, Tokyo 113-0033, Japan.

28 Adler Planetarium, 1300 South Lake Shore Drive, Chicago, IL 60605.

29 Institute for Astronomy, University of Hawaii, 2680 Woodlawn Drive, Honolulu, HI 96822.
} 


\section{INTRODUCTION}

In the last 5 years, the acoustic peaks in the cosmic microwave background (CMB) anisotropy power spectrum have emerged as one of the strongest cosmological probes (Miller et al. 1999; de Bernardis et al. 2000; Hanany et al. 2000; Halverson et al. 2002; Lee et al. 2001; Netterfield et al. 2002; Benoît et al. 2003; Pearson et al. 2003; Bennett et al. 2003). They measure the contents and curvature of the universe (Jungman et al. 1996; Knox \& Page 2000; Lange et al. 2001; Jaffe et al. 2001; Knox et al. 2001; Efstathiou et al. 2002; Percival et al. 2002; Spergel et al. 2003; Tegmark et al. 2004b), demonstrate that the cosmic perturbations are generated early $(z \gg 1000)$ and are dominantly adiabatic $(\mathrm{Hu}$ \& White 1996a, 1996b; Hu et al. 1997a; Peiris et al. 2003; Moodley et al. 2004), and by their mere existence largely validate the simple theory used to support their interpretation (for reviews, see $\mathrm{Hu}$ et al. 1997; Hu \& Dodelson 2002).

The acoustic peaks occur because the cosmological perturbations excite sound waves in the relativistic plasma of the early universe (Peebles \& Yu 1970; Sunyaev \& Zel'dovich 1970; Bond \& Efstathiou 1984, 1987; Holtzmann 1989). The recombination to a neutral gas at redshift $z \approx 1000$ abruptly decreases the sound speed and effectively ends the wave propagation. In the time between the formation of the perturbations and the epoch of recombination, modes of different wavelength can complete different numbers of oscillation periods. This translates the characteristic time into a characteristic length scale and produces a harmonic series of maxima and minima in the anisotropy power spectrum.

Because the universe has a significant fraction of baryons, cosmological theory predicts that the acoustic oscillations in the plasma will also be imprinted onto the late-time power spectrum of the nonrelativistic matter (Peebles \& Yu 1970; Bond \& Efstathiou 1984; Holtzmann 1989; Hu \& Sugiyama 1996; Eisenstein \& Hu 1998). A simple way to understand this is to consider that from an initial point perturbation common to the dark matter and the baryons, the dark matter perturbation grows in place while the baryonic perturbation is carried outward in an expanding spherical wave (Bashinsky \& Bertschinger 2001, 2002). At recombination, this shell is roughly $150 \mathrm{Mpc}$ in radius. Afterward, the combined dark matter and baryon perturbation seeds the formation of largescale structure. Because the central perturbation in the dark matter is dominant compared to the baryonic shell, the acoustic feature is manifested as a small single spike in the correlation function at $150 \mathrm{Mpc}$ separation.

The acoustic signatures in the large-scale clustering of galaxies yield three more opportunities to test the cosmological paradigm with the early-universe acoustic phenomenon: (1) they would provide smoking-gun evidence for our theory of gravitational clustering, notably the idea that large-scale fluctuations grow by linear perturbation theory from $z \sim 1000$ to the present; (2) they would give another confirmation of the existence of dark matter at $z \sim 1000$, since a fully baryonic model produces an effect much larger than observed; and (3) they would provide a characteristic and reasonably sharp length scale that can be measured at a wide range of redshifts, thereby determining purely by geometry the angular diameter-distance-redshift relation and the evolution of the Hubble parameter (Eisenstein et al. 1998b; Eisenstein 2002). The last application can provide precise and robust constraints (Blake \& Glazebrook 2003; Hu \& Haiman 2003; Linder 2003; Seo \& Eisenstein 2003; Amendola et al. 2005; Dolney et al. 2004; Matsubara 2004) on the acceleration of the expansion rate of the universe (Riess et al. 1998; Perlmutter et al. 1999). The nature of the "dark energy" causing this ac- celeration is a complete mystery at present (for a review, see Padmanabhan 2005), but sorting between the various exotic explanations will require superbly accurate data. The acoustic peak method could provide a geometric complement to the usual luminosity-distance methods, such as those based on Type Ia supernovae (e.g., Riess et al. 1998, 2004; Perlmutter et al. 1999; Knop et al. 2003; Tonry et al. 2003).

Unfortunately, the acoustic features in the matter correlations are weak ( $10 \%$ contrast in the power spectrum) and on large scales. This means that one must survey very large volumes, of order $1 h^{-3} \mathrm{Gpc}^{3}$, to detect the signature (Tegmark 1997; Goldberg \& Strauss 1998; Eisenstein et al. 1998b). Previous surveys have not found clean detections, due to sample size and (in some cases) survey geometry. Early surveys (Broadhurst et al. 1990; Landy et al. 1996; Einasto et al. 1997) found anomalous peaks that, although unlikely to be acoustic signatures (Eisenstein et al. 1998a), did not reappear in larger surveys. Percival et al. (2001) favored baryons at $2 \sigma$ in a power spectrum analysis of data from the Two Degree Field Galaxy Redshift Survey (2dFGRS; Colless et al. 2001), but Tegmark et al. (2002) did not recover the signal with $65 \%$ of the same data. Miller et al. (2002) argued that due to smearing from the window function, the 2dFGRS result could only be due to the excess of power on large scales in baryonic models and not to a detection of the oscillations themselves. A spherical harmonic analysis of the full 2dFGRS (Percival et al. 2004) did not find a significant baryon fraction. Miller et al. (2001) presented a "possible detection" $(2.2 \sigma)$ from a combination of three smaller surveys. The analysis of the power spectrum of the Sloan Digital Sky Survey (SDSS) main sample (Tegmark et al. 2004a, 2004b) did not address the question explicitly but would not have been expected to detect the oscillations. The analysis of the correlation function of the 2dFGRS (Hawkins et al. 2003) and the SDSS main sample (Zehavi et al. 2005b) did not consider these scales. Large quasar surveys (Outram et al. 2003; Croom et al. 2004, 2005; Yahata et al. 2005) are too limited by shot noise to reach the required precision, although the high redshift does give leverage on dark energy (Outram et al. 2004) via the Alcock-Paczyński (1979) test. Roukema et al. (2002) attempt to use a quasar sample tracking a comoving feature through redshift as a standard ruler.

In this paper, we present the large-scale correlation function of a large spectroscopic sample of luminous red galaxies (LRGs) from the SDSS (York et al. 2000). This sample covers $3816 \mathrm{deg}^{2}$ out to a redshift of $z=0.47$ with 46,748 galaxies. While it contains fewer galaxies than the 2dFGRS or the main sample of the SDSS, the LRG sample (Eisenstein et al. 2001) has been optimized for the study of structure on the largest scales, and as a result it is expected to significantly outperform those samples. First results on intermediate and small-scale clustering of the LRG sample were presented by Zehavi et al. (2005a) and Eisenstein et al. (2005), and the sample is also being used for the study of galaxy clusters and for the evolution of massive galaxies (Eisenstein et al. 2003). Here we focus on large scales and present the first clear detection of the acoustic peak at late times.

The outline of the paper is as follows. We introduce the SDSS and the LRG sample in $\S 2$. In $\S 3$ we present the LRG correlation function and its covariance matrix, along with a discussion of tests we have performed. In $\S 4$ we fit the correlation function to theoretical models and construct measurements on the cosmological distance scale and cosmological parameters. We conclude in $\S 5$ with a general discussion of the results. Readers wishing to focus on the results rather than the details of the measurement may want to skip $\S \S 3.2,3.3$, and 4.2. 


\section{THE SDSS LUMINOUS RED GALAXY SAMPLE}

The SDSS (York et al. 2000; Stoughton et al. 2002; Abazajian et al. 2003, 2004) is imaging $10^{4} \mathrm{deg}^{2}$ of high Galactic latitude sky in five passbands, $u, g, r, i$, and $z$ (Fukugita et al. 1996; Gunn et al. 1998). Image processing (Lupton et al. 2001; Stoughton et al. 2002; Pier et al. 2003; Ivezić et al. 2004) and calibration (Hogg et al. 2001; Smith et al. 2002) allow one to select galaxies, quasars, and stars for follow-up spectroscopy with twin fiber-fed double spectrographs. Targets are assigned to plug plates with a tiling algorithm that ensures nearly complete samples (Blanton et al. 2003); observing each plate generates 640 spectra covering $3800-9200 \AA$ with a resolution of 1800 .

We select galaxies for spectroscopy by two algorithms. The primary sample (Strauss et al. 2002), referred to here as the SDSS main sample, targets galaxies brighter than $r=17.77$. The surface density of such galaxies is about 90 per square degree, and the median redshift is 0.10 with a tail out to $z \sim 0.25$. The LRG algorithm (Eisenstein et al. 2001) selects $\sim 12$ additional galaxies per square degree, using color-magnitude cuts in $g, r$, and $i$ to select galaxies to a Petrosian magnitude $r<19.5$ that are likely to be luminous early-types at redshifts up to $\sim 0.5$. All fluxes are corrected for extinction (Schlegel et al. 1998) before use. The selection is extremely efficient, and the redshift success rate is very high. A few additional galaxies (three per square degree at $z>$ 0.16 ) matching the rest-frame color and luminosity properties of the LRGs are extracted from the SDSS main sample; we refer to this combined set as the LRG sample.

For our clustering analysis, we use 46,748 luminous red galaxies over $3816 \mathrm{deg}^{2}$ and in the redshift range $0.16-0.47$. The sky coverage of the sample is shown in Hogg et al. (2005) and is similar to that of SDSS Data Release 3 (Abazajian et al. 2004). We require that the galaxies have rest-frame $g$-band absolute magnitudes $-23.2<M_{g}<-21.2\left(h=1, H_{0}=100 h \mathrm{~km} \mathrm{~s}^{-1}\right.$ $\mathrm{Mpc}^{-1}$ ), where we have applied $k$-corrections and passively evolved the galaxies to a fiducial redshift of 0.3 . The resulting comoving number density is close to constant out to $z=0.36$ (i.e., volume-limited) and drops thereafter; see Figure 1 of Zehavi et al. (2005a). The LRG sample is unusual as compared to fluxlimited surveys because it uses the same type of galaxy (luminous early-type galaxies) at all redshifts.

We model the radial and angular selection functions using the methods in the Appendix of Zehavi et al. (2005a). In brief, we build a model of the redshift distribution of the sample by integrating an empirical model of the luminosity function and color distribution of the LRGs with respect to the luminosity-color selection boundaries of the sample. The model is smooth on small scales and includes the subtle interplay of the color-redshift relation and the color selection boundaries. To include slow evolutionary effects, we force the model to the observed redshift histogram using low-pass filtering. We will show in $\S 3.2$ that this filtering does not affect the correlation function on the scales of interest.

The angular selection function is based on the spherical polygon description of 1ss_sample14 (Blanton et al. 2005). We model fiber collisions and unobserved plates by the methods of Zehavi et al. (2005a). Regions with completeness below $60 \%$ (due to unobserved plates) are dropped entirely; the $3816 \mathrm{deg}^{2}$ we use are highly complete. The survey mask excludes regions around bright stars but does not otherwise model small-scale imperfections in the data.

The typical redshift of the sample is $z=0.35$. The coordinate distance to this redshift is $962 h^{-1} \mathrm{Mpc}$ for $\Omega_{m}=0.3, \Omega_{\Lambda}=0.7$. At this distance, $100 h^{-1}$ Mpc subtends $6^{\circ} .0$ on the sky and

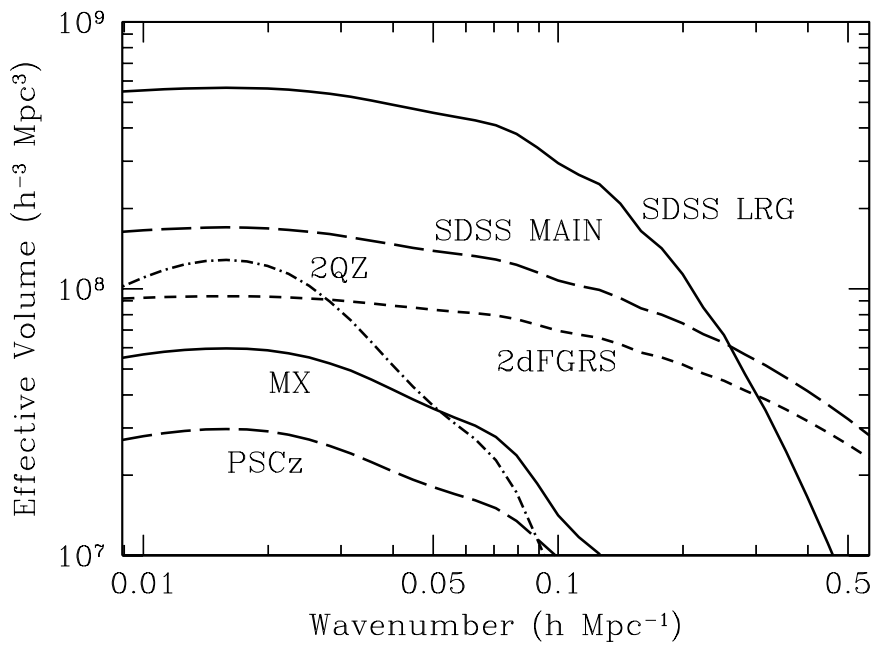

FIG. 1.-Effective volume (eq. [1]) as a function of wavenumber for various large redshift surveys. The effective volume is a rough guide to the performance of a survey (errors scaling as $V_{\text {eff }}^{-1 / 2}$ ) but should not be trusted to better than $30 \%$. To facilitate comparison, we have assumed $3816 \mathrm{deg}^{2}$ for the SDSS main sample, the same area as the SDSS LRG sample presented in this paper and similar to the area in Data Release 3. This is about $50 \%$ larger than the sample analyzed in Tegmark et al. (2004a), which would be similar to the curve for the full $2 \mathrm{dFGRS}$ (Colless et al. 2003). We have neglected the potential gains on very large scales from the 99 outrigger fields of the 2dFGRS. The other surveys are the MX survey of clusters (Miller \& Batuski 2001), the PSCz survey of galaxies (Sutherland et al. 1999), and the 2QZ survey of quasars (Croom et al. 2004). The SDSS DR3 quasar survey (Schneider et al. 2005) is similar in effective volume to the 2QZ. For the amplitude of $P(k)$, we have used $\sigma_{8}=1$ for 2QZ and PSCz and 3.6 for the MX survey. We used $\sigma_{8}=1.8$ for SDSS LRG, SDSS main, and the 2dFGRS. For the latter two, this value represents the amplitude of clustering of the luminous galaxies at the surveys' edge; at lower redshift, the number density is so high that the choice of $\sigma_{8}$ is irrelevant. Reducing SDSS main or 2dFGRS to $\sigma_{8}=1$, the value typical of normal galaxies, decreases their $V_{\text {eff }}$ by $30 \%$. [See the electronic edition of the Journal for a color version of this figure.]

corresponds to 0.04 in redshift. The LRG correlation length of $10 h^{-1} \mathrm{Mpc}$ (Zehavi et al. 2005a) subtends only $40^{\prime}$ on the sky at $z=0.35$; viewed on this scale, the survey is far more bulk than boundary.

A common way to assess the statistical reach of a survey, including the effects of shot noise, is by the effective volume (Feldman et al. 1994; Tegmark 1997)

$$
V_{\mathrm{eff}}(k)=\int d^{3} r\left[\frac{n(\boldsymbol{r}) P(k)}{1+n(\boldsymbol{r}) P(k)}\right]^{2},
$$

where $n(\boldsymbol{r})$ is the comoving number density of the sample at every location $\boldsymbol{r}$. The effective volume is a function of the wavenumber $k$ via the power amplitude $P$. For $P=10^{4} h^{-3} \mathrm{Mpc}^{3}(k \approx$ $0.15 h \mathrm{Mpc}^{-1}$ ), we find $0.13 h^{-3} \mathrm{Gpc}^{3}$; for $P=4 \times 10^{4} h^{-3} \mathrm{Mpc}^{3}$ $\left(k \approx 0.05 h \mathrm{Mpc}^{-1}\right), 0.38 h^{-3} \mathrm{Gpc}^{3}$; and for $P=10^{5} h^{-3} \mathrm{Mpc}^{3}$ $\left(k \approx 0.02 h \mathrm{Mpc}^{-1}\right), 0.55 h^{-3} \mathrm{Gpc}^{3}$. The actual survey volume is $0.72 h^{-3} \mathrm{Gpc}^{3}$, so there are roughly 700 cubes of $100 h^{-1} \mathrm{Mpc}$ size in the survey. The relative sparseness of the LRG sample, $n \sim 10^{-4} h^{3} \mathrm{Mpc}^{-3}$, is well suited to measuring power on large scales (Kaiser 1986).

In Figure 1 we show a comparison of the effective volume of the SDSS LRG survey to other published surveys. While this calculation is necessarily a rough $(\sim 30 \%)$ predictor of statistical performance due to neglect of the exact survey boundary and our detailed assumptions about the amplitude of the power spectrum and the number density of objects for each survey, the SDSS LRG is clearly the largest survey to date for studying the linear 
regime by a factor of $\sim 4$. The LRG sample should therefore outperform these surveys by a factor of 2 in fractional errors on large scales. Note that quasar surveys cover much more volume than even the LRG survey, but their effective volumes are worse, even on large scales, due to shot noise.

\section{THE REDSHIFT-SPACE CORRELATION FUNCTION}

\subsection{Correlation Function Estimation}

In this paper, we analyze the large-scale clustering using the two-point correlation function (Peebles 1980, § 71). In recent years, the power spectrum has become the common choice on large scales, as the power in different Fourier modes of the linear density field is statistically independent in standard cosmology theories (Bardeen et al. 1986). However, this advantage breaks down on small scales due to nonlinear structure formation, while on large scales elaborate methods are required to recover the statistical independence in the face of survey boundary effects (for discussion, see Tegmark et al. 1998). The power spectrum and correlation function contain the same information in principle, as they are Fourier transforms of one another. The property of the independence of different Fourier modes is not lost in real space, but rather it is encoded into the off-diagonal elements of the covariance matrix via a linear basis transformation. One must therefore accurately track the full covariance matrix to use the correlation function properly, but this is feasible. An advantage of the correlation function is that, unlike in the power spectrum, small-scale effects such as shot noise and intrahalo astrophysics stay on small scales, well separated from the linear regime fluctuations and acoustic effects.

We compute the redshift-space correlation function using the Landy-Szalay estimator (Landy \& Szalay 1993). Random catalogs containing at least 16 times as many galaxies as the LRG sample were constructed according to the radial and angular selection functions described above. We assume a flat cosmology with $\Omega_{m}=0.3$ and $\Omega_{\Lambda}=0.7$ when computing the correlation function. We place each data point in its comoving coordinate location based on its redshift and compute the comoving separation between two points using the vector difference. We use bins in separations of $4 h^{-1} \mathrm{Mpc}$ from 10 to $30 h^{-1} \mathrm{Mpc}$ and bins of $10 h^{-1} \mathrm{Mpc}$ thereafter out to $180 h^{-1} \mathrm{Mpc}$, for a total of 20 bins.

We weight the sample using a scale-independent weighting that depends on redshift. When computing the correlation function, each galaxy and random point is weighted by $1 /\left[1+n(z) P_{w}\right]$ (Feldman et al. 1994), where $n(z)$ is the comoving number density and $P_{w}=40,000 h^{-3} \mathrm{Mpc}^{3}$. We do not allow $P_{w}$ to change with scale so as to avoid scale-dependent changes in the effective bias caused by differential changes in the sample redshift. Our choice of $P_{w}$ is close to optimal at $k \approx 0.05 h \mathrm{Mpc}^{-1}$ and within $5 \%$ of the optimal errors for all scales relevant to the acoustic oscillations $\left(k \lesssim 0.15 h \mathrm{Mpc}^{-1}\right)$. At $z<0.36, n P_{w}$ is about 4 , while $n P_{w} \approx 1$ at $z=0.47$. Our results do not depend on the value of $P_{w}$; changing the value wildly alters our best-fit results by only $0.1 \sigma$.

Redshift distortions cause the redshift-space correlation function to vary according to the angle between the separation vector and the line of sight. To ease comparison to theory, we focus on the spherically averaged correlation function. Because of the boundary of the survey, the number of possible tangential separations is somewhat underrepresented compared to the number of possible line-of-sight separations, particularly at very large scales. To correct for this, we compute the correlation functions in four angular bins. The effects of redshift distortions are obvious: large-separation correlations are smaller along the line-of-

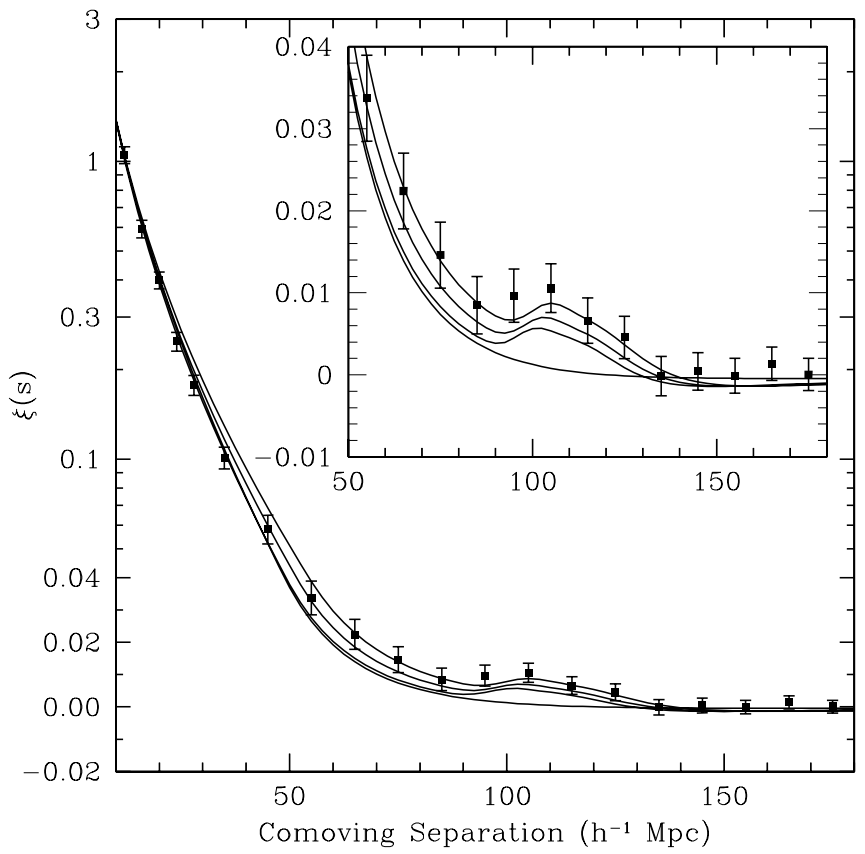

FIG. 2.- Large-scale redshift-space correlation function of the SDSS LRG sample. The error bars are from the diagonal elements of the mock-catalog covariance matrix; however, the points are correlated. Note that the vertical axis mixes logarithmic and linear scalings. The inset shows an expanded view with a linear vertical axis. The models are $\Omega_{m} h^{2}=0.12$ (top line), 0.13 (second line), and 0.14 (third line), all with $\Omega_{b} h^{2}=0.024$ and $n=0.98$ and with a mild nonlinear prescription folded in. The bottom line shows a pure CDM model $\left(\Omega_{m} h^{2}=\right.$ $0.105)$, which lacks the acoustic peak. It is interesting to note that although the data appear higher than the models, the covariance between the points is soft as regards overall shifts in $\xi(s)$. Subtracting 0.002 from $\xi(s)$ at all scales makes the plot look cosmetically perfect but changes the best-fit $\chi^{2}$ by only 1.3 . The bump at $100 h^{-1} \mathrm{Mpc}$ scale, on the other hand, is statistically significant. [See the electronic edition of the Journal for a color version of this figure.]

sight direction than along the tangential direction. We sum these four correlation functions in the proportions corresponding to the fraction of the sphere included in the angular bin, thereby recovering the spherically averaged redshift-space correlation function. We have not yet explored the cosmological implications of the anisotropy of the correlation function (Matsubara \& Szalay 2003).

The resulting redshift-space correlation function is shown in Figure 2. A more convenient view is shown in Figure 3, where we have multiplied by the square of the separation, so as to flatten out the result. The errors and overlaid models will be discussed below. The bump at $100 h^{-1} \mathrm{Mpc}$ is the acoustic peak, to be described in $\S 4.1$.

The clustering bias of LRGs is known to be a strong function of luminosity (Hogg et al. 2003; Eisenstein et al. 2005; Zehavi et al. 2005a), and while the LRG sample is nearly volume-limited out to $z \sim 0.36$, the flux cut does produce a varying luminosity cut at higher redshifts. If larger scale correlations were preferentially drawn from higher redshift, we would have a differential bias (see discussion in Tegmark et al. 2004a). However, Zehavi et al. (2005a) have studied the clustering amplitude in the two limiting cases, namely the luminosity threshold at $z<0.36$ and that at $z=0.47$. The differential bias between these two samples on large scales is modest, only $15 \%$. We make a simple parameterization of the bias as a function of redshift and then compute $b^{2}$ averaged as a function of scale over the pair counts in the random catalog. The bias varies by less than $0.5 \%$ as a function of scale, and so we conclude that there is no effect of a possible correlation of scale with redshift. This test also shows that the 


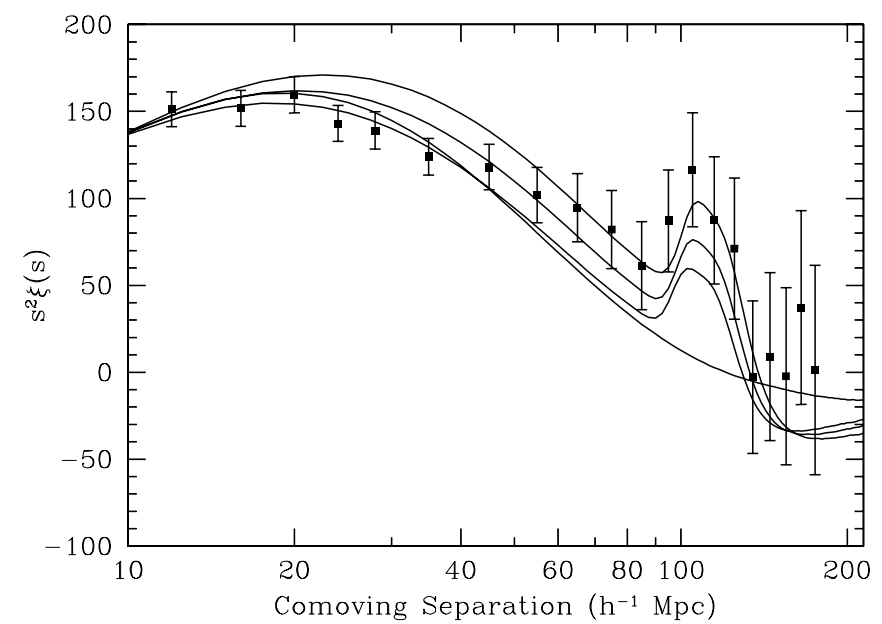

FIG. 3.- Same as Fig. 2, but plotting the correlation function times $s^{2}$. This shows the variation of the peak at $20 h^{-1} \mathrm{Mpc}$ scales that is controlled by the redshift of equality (and hence by $\Omega_{m} h^{2}$ ). Varying $\Omega_{m} h^{2}$ alters the amount of large-to-small scale correlation, but boosting the large-scale correlations too much causes an inconsistency at $30 \mathrm{~h}^{-1} \mathrm{Mpc}$. The pure CDM model (bottom line) is actually close to the best fit due to the data points on intermediate scales. [See the electronic edition of the Journal for a color version of this figure.]

mean redshift as a function of scale changes so little that variations in the clustering amplitude at fixed luminosity as a function of redshift are negligible.

\subsection{Tests for Systematic Errors}

We have performed a number of tests searching for potential systematic errors in our correlation function. First, we have tested that the radial selection function is not introducing features into the correlation function. Our selection function involves smoothing the observed histogram with a boxcar smoothing of width $\Delta z=0.07$. This corresponds to reducing power in the purely radial mode at $k=0.03 h \mathrm{Mpc}^{-1}$ by $50 \%$. Purely radial power at $k=0.04(0.02) h \mathrm{Mpc}^{-1}$ is reduced by $13 \%(86 \%)$. The effect of this suppression is negligible, only $5 \times 10^{-4}\left(10^{-4}\right)$ on the correlation function at the $30(100) h^{-1} \mathrm{Mpc}$ scale. Simply put, purely radial modes are a small fraction of the total at these wavelengths. We find that an alternative radial selection function, in which the redshifts of the random catalog are simply picked randomly from the observed redshifts, produces a negligible change in the correlation function. This of course corresponds to complete suppression of purely radial modes.

The selection of LRGs is highly sensitive to errors in the photometric calibration of the $g, r$, and $i$ bands (Eisenstein et al. 2001). We assess these by making a detailed model of the distribution in color and luminosity of the sample, including photometric errors, and then computing the variation of the number of galaxies accepted at each redshift with small variations in the LRG sample cuts. A $1 \%$ shift in the $r-i$ color makes a $8 \%-10 \%$ change in number density; a $1 \%$ shift in the $g-r$ color makes a $5 \%$ change in number density out to $z=0.41$, dropping thereafter; and a $1 \%$ change in all magnitudes together changes the number density by $2 \%$ out to $z=0.36$, increasing to $3.6 \%$ at $z=0.47$. These variations are consistent with the changes in the observed redshift distribution when we move the selection boundaries to restrict the sample. Such photometric calibration errors would cause anomalies in the correlation function as the square of the number density variations, as this noise source is uncorrelated with the true sky distribution of LRGs.

Assessments of calibration errors based on the color of the stellar locus find only $1 \%$ scatter in $g, r$, and $i$ (Ivezić et al. 2004), which would translate to about 0.02 in the correlation function. However, the situation is more favorable, because the coherence scale of the calibration errors is limited by the fact that the SDSS is calibrated in regions about 0.6 wide and up to $15^{\circ}$ long. This means that there are 20 independent calibrations being applied to a given $6^{\circ}\left(100 h^{-1} \mathrm{Mpc}\right)$ radius circular region. Moreover, some of the calibration errors are even more localized, being caused by small mischaracterizations of the point-spread function and errors in the flat-field vectors early in the survey (Stoughton et al. 2002). Such errors will average down on larger scales even more quickly.

The photometric calibration of the SDSS has evolved slightly over time (Abazajian et al. 2003), but of course the LRG selection was based on the calibrations at the time of targeting. We make our absolute magnitude cut on the latest ("uber") calibrations (Blanton et al. 2005; Finkbeiner et al. 2004), although this is only important at $z<0.36$ as the targeting flux cut limits the sample at higher redshift. We test whether changes in the calibrations might alter the correlation function by creating a new random catalog in which the differences in each band between the target-epoch photometry and the uber-calibration values are mapped to angularly dependent redshift distributions using the number density derivatives presented above. Using this random catalog makes negligible differences in the correlation function on all scales. This is not surprising: while an rms error of $2 \%$ in $r-i$ would give rise to a 0.04 excess in the correlation function, scales large enough that this would matter have many independent calibrations contributing to them. On the other hand, the uber-calibration of the survey does not necessarily fix all calibration problems, particularly variations within a single survey run, and so this test cannot rule out an arbitrary calibration problem.

We continue our search for calibration errors by breaking the survey into 10 radial pieces and measuring the cross-correlations between the nonadjacent slabs. Calibration errors would produce significant correlations on large angular scales. Some crosscorrelations have amplitudes of $2 \%-3 \%$, but many others do not, suggesting that this is simply noise. We also take the full matrix of cross-correlations at a given separation and attempt to model it (minus the diagonal and first off-diagonal elements) as an outer product of vector with itself, as would be appropriate if it were dominated by a single type of radial perturbation, but we do not find plausible or stable vectors, again indicative of noise. Hence, we conclude that systematic errors in $\xi(r)$ due to calibration must be below 0.01 .

It is important to note that calibration errors in the SDSS produce large-angle correlations only along the scan direction. Even if errors were noticeably large, they would not produce narrow features such as that seen at the $100 h^{-1} \mathrm{Mpc}$ scale for two reasons. First, the projection from the three-dimensional sphere to one-dimensional strips on the sky necessarily means that a given angular scale maps to a wide range of three-dimensional separations. Second, the comoving angular diameter distance used to translate angles into transverse comoving separations varies by a factor of 3 from $z=0.16$ to 0.47 , so that a preferred angle would not map to a narrow range of physical scales. We therefore expect that calibration errors would appear as a smooth anomalous correlation, rolling off toward large scales.

Breaking the sample into two redshift slices above and below $z=0.36$ yields similar correlation functions (Fig. 4). Errors in calibration or in the radial selection function would likely enter the two redshift slices in different manners, but we see no sign of this. In particular, the bump in the correlation function appears in both slices. While this could in principle give additional leverage 


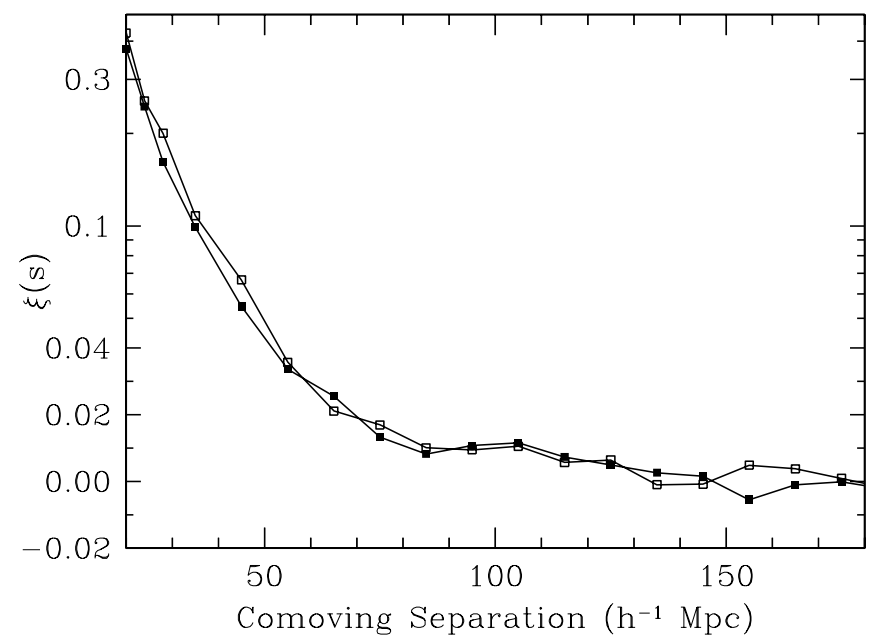

FIG. 4.-Correlation function for two different redshift slices, $0.16<z<$ 0.36 (line with filled squares) and $0.36<z<0.47$ (line with open squares). The latter is somewhat noisier, but the two are quite similar and both show evidence of the acoustic peak. The differences are within the expected statistical variations, as tested by $\chi^{2}$. Note that the vertical axis mixes logarithmic and linear scalings. [See the electronic edition of the Journal for a color version of this figure.]

on the cosmological distance scale, we have not pursued this in this paper.

\subsection{Covariance Matrix}

Because of the large number of separation bins and the large scales being studied, it is infeasible to use jackknife sampling to construct a covariance matrix for $\xi(r)$. Instead, we use a large set of mock catalogs to construct a covariance matrix and then test that matrix with a smaller number of jackknife samples.

Our mock catalogs are constructed using PTHALOS (Scoccimarro \& Sheth 2002) with a halo occupation model (Ma \& Fry 2000; Seljak 2000; Peacock \& Smith 2000; Scoccimarro et al. 2001; Cooray \& Sheth 2002; Berlind et al. 2003) that matches the observed amplitude of clustering of LRGs (Zehavi et al. 2005a). PTHALOS distributes dark matter halos according to extended Press-Schechter theory conditioned by the largescale density field from second-order Lagrangian perturbation theory. This generates density distributions that fully include Gaussian linear theory but also include second-order clustering, redshift distortions, and the small-scale halo structure that dominates the non-Gaussian signal. We use a cosmology of $\Omega_{m}=$ $0.3, \Omega_{\Lambda}=0.7$, and $h=0.7$ for the mock catalogs. We subsample the catalogs so as to match the comoving density of LRGs as a function of redshift exactly, but do not attempt to include the small redshift dependence in the amplitude of the bias. Our catalogs match the angular geometry of the survey except in some fine details involving less than $1 \%$ of the area. Two simulation boxes, each $\left(1250 h^{-1} \mathrm{Mpc}\right)^{2} \times 2500 h^{-1} \mathrm{Mpc}$, one for the north Galactic cap and the other for the south, are pasted together in each catalog. These two regions of the survey are very well separated in space, so this division is harmless. We generate 1278 mock catalogs with independent initial conditions, compute the correlation function in each, and compute the covariance matrix from the variations between them.

The resulting matrix shows considerable correlations between neighboring bins, but with an enhanced diagonal due to shot noise. The power on scales below $10 h^{-1} \mathrm{Mpc}$ creates significant correlation between neighboring scales. A curious aspect is that on large scales, where our bins are $10 h^{-1} \mathrm{Mpc}$ wide, the reduced inverse covariance matrix is quite close to tri-diagonal; the first off-diagonal is typically around 0.4 , but the second and subsequent are typically a few percent. Such matrices correspond to exponential decays of the off-diagonal correlations (Rybicki \& Press 1994).

We test the covariance matrix in several ways. First, as described in $\S 4.3$, the best-fit cosmological model has $\chi^{2}=16.1$ on 17 degrees of freedom ( $p=0.52$ ), indicating that the covariances are of the correct scale. Next, we subdivide the survey into 10 large, compact subregions and look at the variations between jackknifed samples (i.e., computing $\xi$ while excluding one region at a time). The variations of $\xi(r)$ between the 10 jackknife samples match the diagonal elements of the covariance matrix to within $10 \%$. We then test the off-diagonal terms by asking whether the jackknife residuals vary appropriately relative to the covariance matrix. If one defines the residual $\Delta \xi_{j}$ between the $j$ th jackknife sample and the mean, then the construction $\sum_{a b} \Delta \xi_{j}\left(r_{a}\right) C_{a b}^{-1} \Delta \xi_{j}\left(r_{b}\right)$, where $C$ is the covariance matrix and the sums are over the 20 radial bins, should have a mean (averaged over the 10 jackknife sample) of about $20 /(10-1) \approx 2.2$. The mean value is $2.7 \pm 0.5$, in reasonable agreement.

We get similar results for cosmological parameters when using a covariance matrix that is based on constructing the Gaussian approximation (Feldman et al. 1994; Tegmark 1997) of independent modes in Fourier space. We use the effective volume at each wavenumber, plus an extra shot noise term to represent nonGaussian halos, and then rotate this matrix from the diagonal Fourier basis into the real-space basis. This matrix gives reasonable $\chi^{2}$ values, satisfies the jackknife tests, and gives similar values and errors on cosmological parameters.

Finally, we test our results for cosmological parameters by using the following hybrid scheme. We use the mock-catalog covariance matrix to find the best-fit cosmological model for each of the 10 jackknife samples, and then use the rms of the 10 bestfit parameter sets to determine the errors. This means that we are using the mock catalogs to weight the $\xi(r)$ measurements, but relying on the jackknifed samples to actually determine the variance on the cosmological parameters. We will quote the numerical results in $\S 4.3$, but here we note that the resulting constraints on the acoustic scale and matter density match the errors inferred from the fitting with the mock-catalog covariance matrix. Hence, we conclude that our covariance matrix is generating correct results for our model fitting and cosmological parameter estimates.

\section{CONSTRAINTS ON COSMOLOGICAL MODELS}

\subsection{Linear Theory}

Given the value of the matter density $\Omega_{m} h^{2}$, the baryon density $\Omega_{b} h^{2}$, the spectral tilt $n$, and a possible neutrino mass, adiabatic cold dark matter (CDM) models predict the linear matter power spectrum (and correlation function) up to an amplitude factor. There are two primary physical scales at work (for discussions, see $\mathrm{Hu}$ et al. 1997a; Eisenstein \& Hu 1998; Hu \& Dodelson 2002). First, the clustering of CDM is suppressed on scales small enough to have been traversed by the neutrinos and/or photons during the radiation-dominated period of the universe. This introduces the characteristic turnover in the CDM power spectrum at the scale of the horizon at matter-radiation equality. This length scales as $\left(\Omega_{m} h^{2}\right)^{-1}$ (assuming the standard cosmic neutrino background). Second, the acoustic oscillations have a characteristic scale known as the sound horizon, which is the comoving distance that a sound wave can travel between the big bang and recombination. This depends both on the expansion history of 
the early universe and on the sound speed in the plasma; for models close to the concordance cosmology, this length scales as $\left(\Omega_{m} h^{2}\right)^{-0.25}\left(\Omega_{b} h^{2}\right)^{-0.08}$ (Hu 2005). The spectral tilt and massive neutrinos tilt the low-redshift power spectrum (Bond \& Szalay 1983) but do not otherwise change these two scales. Importantly, given $\Omega_{m} h^{2}$ and $\Omega_{b} h^{2}$, the two physical scales are known in absolute units, with no factors of $H_{0}^{-1}$.

We use CMBFAST (Seljak \& Zaldarriaga 1996; Zaldarriaga et al. 1998; Zaldarriaga \& Seljak 2000) to compute the linear power spectra, which we convert to correlation functions with a Fourier transform. It is important to note that the harmonic series of acoustic peaks found in the power spectrum transform to a single peak in the correlation function (Matsubara 2004). The decreasing envelope of the higher harmonics, due to Silk damping (Silk 1968) or nonlinear gravity (Meiksin et al. 1999), corresponds to a broadening of the single peak. This broadening decreases the accuracy with which one can measure the centroid of the peak, equivalent to the degradation of acoustic scale measurements caused by the disappearance of higher harmonics in the power spectrum (Seo \& Eisenstein 2003).

Examples of the model correlation functions are shown in Figures 2 and 3, each with $\Omega_{b} h^{2}=0.024$ and $h=0.7$ but with three values of $\Omega_{m} h^{2}$. Higher values of $\Omega_{m} h^{2}$ correspond to earlier epochs of matter-radiation equality, which increase the amount of small-scale power compared to large, which in turn decreases the correlations on large scales when holding the small-scale amplitude fixed. The acoustic scale, on the other hand, depends only weakly on $\Omega_{m} h^{2}$.

\subsection{Nonlinear Corrections}

The precision of the LRG correlations is such that we cannot rely entirely on linear theory even at $r>10 h^{-1} \mathrm{Mpc}$ (wavenumbers $k<0.1 h \mathrm{Mpc}^{-1}$ ). Nonlinear gravity (Meiksin et al. 1999), redshift distortions (Kaiser 1987; Hamilton 1998; Scoccimarro 2004), and scale-dependent bias all enter at a subtle level. We address these by applying corrections derived from $\mathrm{N}$-body simulations and the Smith et al. (2003) halofit method.

Seo \& Eisenstein (2005) present a set of $51 \mathrm{~N}$-body simulations of the Wilkinson Microwave Anisotropy Probe (WMAP) best-fit cosmology (Spergel et al. 2003). Each simulation has $256^{3}$ particles and is $512 h^{-1} \mathrm{Mpc}$ in size. The outputs at $z=0.3$ were analyzed to find their power spectra and correlation functions, in both real and redshift space, including simple halo bias. This provides an accurate description of the nonlinear gravitational and redshift distortion effects on large scales.

For a general cosmology, we begin from the CMBFAST linear power spectrum. We next correct for the erasure of the higher acoustic peak that occurs due to mode coupling (Meiksin et al. 1999). We do this by generating the "no wiggle" approximation from Eisenstein \& Hu (1998), which matches the overall shape of the linear power spectrum but with the acoustic oscillations edited out, and then smoothly interpolating between the linear spectrum and the approximate one. We use a Gaussian exp $\left[-(k a)^{2}\right]$, with a scale $a=7 h^{-1} \mathrm{Mpc}$ chosen to approximately match the suppression of the oscillations seen in the power spectrum of the $N$-body simulations.

We next use the Smith et al. (2003) package to compute the alterations in power from nonlinear gravitational collapse. We use a model with $\Gamma=0.162$ and $\sigma_{8}=0.85$. This model has the same shape for the power spectrum on small scales as the WMAP best-fit cosmology and is therefore a reasonable zero-baryon model with which to compute. We find the quotient of the nonlinear and linear power spectra for this model and multiply that ratio onto our baryonic power spectrum. We then Fourier trans-

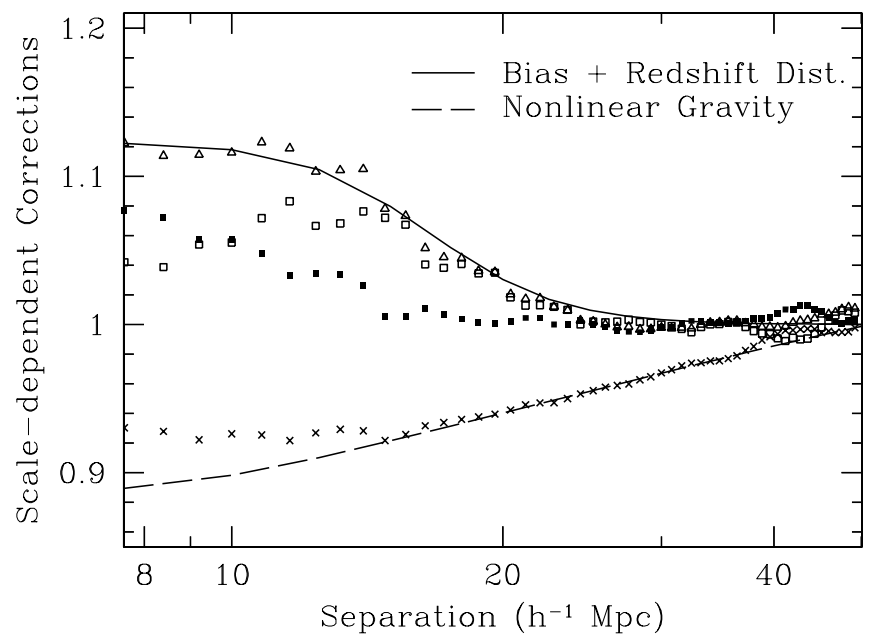

Fig. 5.-Scale-dependent corrections derived from $51 \mathrm{~N}$-body simulations, each $512 h^{-1} \mathrm{Mpc}$ comoving with $256^{3}$ particles (Seo \& Eisenstein 2005). The crosses show the ratio between the nonlinear matter correlation function and the linear correlation function; the dashed line is the model we use from Smith et al. (2003). The solid points are the ratio of the biased correlation function (using a simple halo mass cut) to the nonlinear matter correlation function. The open squares are the ratio of the biased redshift-space correlation function to the biased real-space correlation function, after removing the large-scale asymptotic value (Kaiser 1987), which we simply fold into the correlation amplitude parameter. The open triangles show the product of these two effects, and the solid line is our fit to this product. These corrections are of order $10 \%$ at $10 h^{-1} \mathrm{Mpc}$ separations and decrease quickly on larger scales. In addition to these corrections, we mimic the erasure of the small-scale acoustic oscillations in the power spectrum by using a smoothed crossover at $k=0.14 h \mathrm{Mpc}^{-1}$ between the CMBFAST linear power spectrum and the no-wiggle form from Eisenstein \& $\mathrm{Hu}$ (1998).

form the power spectrum to generate the real-space correlation function. The accuracy of this correction is shown in the bottom of Figure 5: the crosses show the ratio in the $\mathrm{N}$-body simulations between the $z=0.3$ and $z=49$ correlation functions, while the dashed line shows the Smith et al. (2003) derived correction.

Next we correct for redshift distortions and halo bias. We use the $N$-body simulations to find the ratio of the redshift-space biased correlation function to the real-space matter correlation function for a halo mass threshold that approximately matches the observed LRG clustering amplitude. This ratio approaches an asymptotic value on large scales that we simply include into the large-scale clustering bias. After removing this asymptotic value, the remaining piece of the ratio is only $10 \%$ at $r=$ $10 h^{-1} \mathrm{Mpc}$. We fit this to a simple smooth function and then multiply the model correlation functions by this fit. Figure 5 shows the $N$-body results for the ratio of redshift-space $\xi$ to realspace $\xi$ for the halos and the ratio of the real-space halo correlation function to that of the matter. The solid line shows our fit to the product. Tripling the mass threshold of the bias model increases the correction by only $30 \%$ (i.e., $1.12-1.16$ on small scales).

All of the corrections in Figure 5 are clearly small, only $10 \%$ at $r>10 h^{-1} \mathrm{Mpc}$. This is because $10 h^{-1} \mathrm{Mpc}$ separations are larger than any virialized halo and are only affected by the extremes of the finger-of-God redshift distortions. While our methods are not perfect, they are plausibly matched to the allowed cosmology and to the bias of the LRGs. As such, we believe that the corrections should be accurate to a few percent, which is sufficient for our purposes. Similarly, while we have derived the corrections for a single cosmological model, the data constrain the allowed cosmologies enough that variations in the corrections will be smaller than our tolerances. For example, increasing 
$\sigma_{8}$ to 1.0 for the halofit calculation changes the corrections at $r>10 h^{-1} \mathrm{Mpc}$ by less than $2 \%$.

We stress that while galaxy clustering bias does routinely affect large-scale clustering (obviously so in the LRG sample, with bias $b \approx 2$ ), it is very implausible that it would mimic the acoustic signature, as this would require galaxy formation physics to have a strong preferred scale at $100 \mathrm{~h}^{-1} \mathrm{Mpc}$. Galaxy formation prescriptions that involve only small-scale physics, such as that involving dark matter halos or even moderate-scale radiation transport, necessarily produce smooth effects on large scales (Coles 1993; Fry \& Gaztanaga 1993; Scherrer \& Weinberg 1998). Even long-range effects that might be invoked would need to affect $100 h^{-1} \mathrm{Mpc}$ scales differently from 80 or 130 . Our detection of the acoustic peak cannot reasonably be explained as an illusion of galaxy formation physics.

\subsection{Measurements of the Acoustic and Equality Scales}

The observed LRG correlation function could differ from that of the correct cosmological model in amplitude, because of clustering bias and uncertain growth functions, and in scale, because we may have used an incorrect cosmology in converting from redshift into distance. Our goal is to use the comparison between observations and theory to infer the correct distance scale.

Note that in principle a change in the cosmological model would change the distances differently for different redshifts, requiring us to recompute the correlation function for each model choice. In practice, the changes are small enough and the redshifts close enough that we treat the variation as a single dilation in scale (similar to Blake \& Glazebrook 2003). This would be a superb approximation at low redshift, where all distances behave inversely with the Hubble constant. By $z=0.35$, the effects of cosmological acceleration are beginning to enter. However, we have checked explicitly that our single-scale approximation is good enough for $\Omega_{m}$ between 0.2 and 0.4 . Relative to our fiducial scale at $z=0.35$, the change in distance across the redshift range $0.16<$ $z<0.47$ is only $3 \%$ peak to peak for $\Omega_{m}=0.2$ compared to 0.3 , and even these variations largely cancel around the $z=0.35$ midpoint where we will quote our cosmological constraints.

The other error in our one scale parameter approximation is to treat the line-of-sight dilation equivalently to the transverse dilation. In truth, the Hubble parameter changes differently from the angular diameter distance (the Alcock-Paczyński [1979] effect). For small deviations from $\Omega_{m}=0.3$ and $\Omega_{\Lambda}=0.7$, the change in the Hubble parameter at $z=0.35$ is about half that of the angular diameter distance. We model this by treating the dilation scale as the cube root of the product of the radial dilation times the square of the transverse dilation. In other words, we define

$$
D_{V}(z)=\left[D_{M}(z)^{2} \frac{c z}{H(z)}\right]^{1 / 3},
$$

where $H(z)$ is the Hubble parameter and $D_{M}(z)$ is the comoving angular diameter distance. As the typical redshift of the sample is $z=0.35$, we quote our result for the dilation scale as $D_{V}(0.35)$. For our fiducial cosmology of $\Omega_{m}=0.3, \Omega_{\Lambda}=0.7$, and $h=$ $0.7, D_{V}(0.35)=1334 \mathrm{Mpc}$.

We compute parameter constraints by computing $\chi^{2}$ (using the full covariance matrix) for a grid of cosmological models. In addition to cosmological parameters of $\Omega_{m} h^{2}, \Omega_{b} h^{2}$, and $n$, we include the distance scale $D_{V}(0.35)$ of the LRG sample and marginalize over the amplitude of the correlation function. Param-

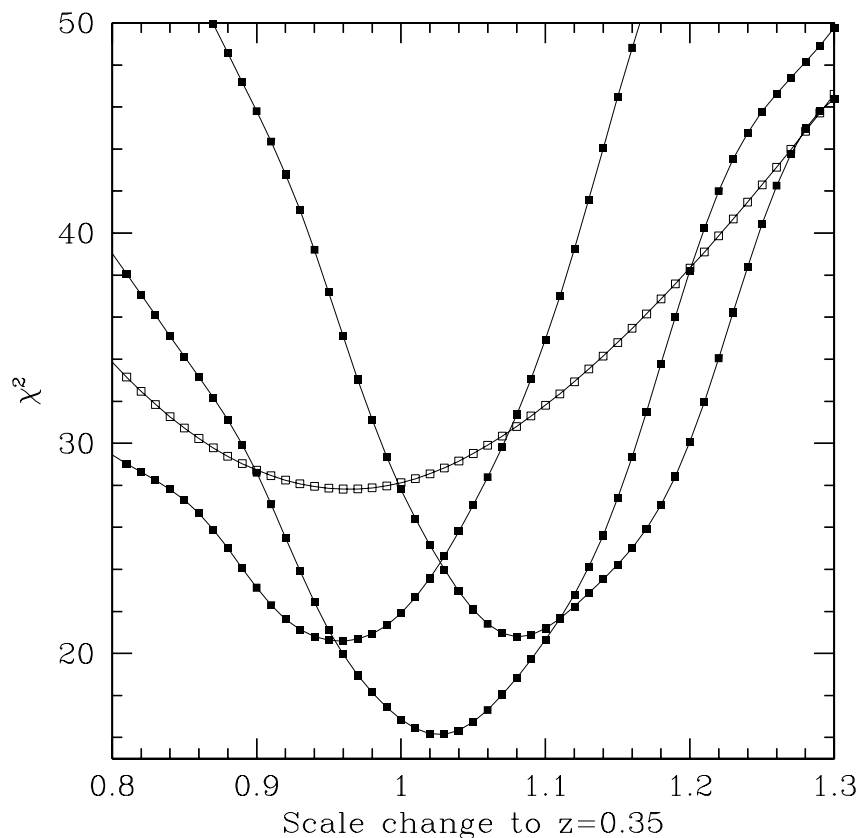

FIG. 6.-The $\chi^{2}$ values of the models as a function of the dilation of the scale of the correlation function. This corresponds to altering $D_{V}(0.35)$ relative to the baseline cosmology of $\Omega=0.3, \Omega_{\Lambda}=0.7$, and $h=0.7$. Each line (save the line with open squares) in the plot is a different value of $\Omega_{m} h^{2}, 0.11,0.13$, and 0.15 from left to right; $\Omega_{b} h^{2}=0.024$ and $n=0.98$ are used in all cases. The amplitude of the model has been marginalized over. The best-fit $\chi^{2}$ is 16.1 on 17 degrees of freedom, consistent with expectations. The line with open squares shows the pure CDM model with $\Omega_{m} h^{2}=0.10$; it has a best $\chi^{2}$ of 27.8, which is rejected at $3.4 \sigma$. Note that this curve is also much broader, indicating that the lack of an acoustic peak makes the scale less constrainable. [See the electronic edition of the Journal for a color version of this figure.]

eters such as $h, \Omega_{m}, \Omega_{K}$, and $w(z)$ are subsumed within $D_{V}(0.35)$. We assume $h=0.7$ when computing the scale at which to apply the nonlinear corrections; having set those corrections, we then dilate the scale of the final correlation function.

The WMAP data (Bennett et al. 2003), as well as combinations of WMAP with large-scale structure (Spergel et al. 2003; Tegmark et al. 2004b), the Ly $\alpha$ forest (McDonald et al. 2004; Seljak et al. 2005), and big bang nucleosynthesis (e.g., Burles et al. 2001; Coc et al. 2004), constrain $\Omega_{b} h^{2}$ and $n$ rather well, and so to begin, we hold these parameters fixed (at 0.024 and 0.98 , respectively), and consider only variations in $\Omega_{m} h^{2}$. In practice, the sound horizon varies only as $\left(\Omega_{b} h^{2}\right)^{-0.08}$, which means that the tight constraints from WMAP (Spergel et al. 2003) and big bang nucleosynthesis (Burles et al. 2001) make the uncertainties in the baryon density negligible.

Figure 6 shows $\chi^{2}$ as a function of the dilation for three different values of $\Omega_{m} h^{2}, 0.11,0.13$, and 0.15 . Scanning across all $\Omega_{m} h^{2}$, the best-fit $\chi^{2}$ is 16.1 on 17 degrees of freedom [20 data points and three parameters: $\Omega_{m} h^{2}, D_{V}(0.35)$, and the amplitude]. Figure 7 shows the contours of equal $\chi^{2}$ in $\Omega_{m} h^{2}$ and $D_{V}(0.35)$, corresponding to $1 \sigma$ up to $5 \sigma$ for a two-dimensional Gaussian likelihood function. Adopting a likelihood proportional to $\exp \left(-\chi^{2} / 2\right)$, we project the axes to find $\Omega_{m} h^{2}=0.130 \pm 0.010$ and $D_{V}(0.35)=1370 \pm 64 \mathrm{Mpc}(4.7 \%)$, where these are $1 \sigma$ errors.

Figure 7 also contains two lines that depict the two physical scales. The solid line is that of constant $\Omega_{m} h^{2} D_{V}$, which would place the (matter-radiation) equality scale at a constant apparent location. This would be the degeneracy direction for a pure CDM cosmology and would be a line of constant $\Gamma=\Omega_{m} h$ were the LRG sample at lower redshift. The dashed line holds constant the 


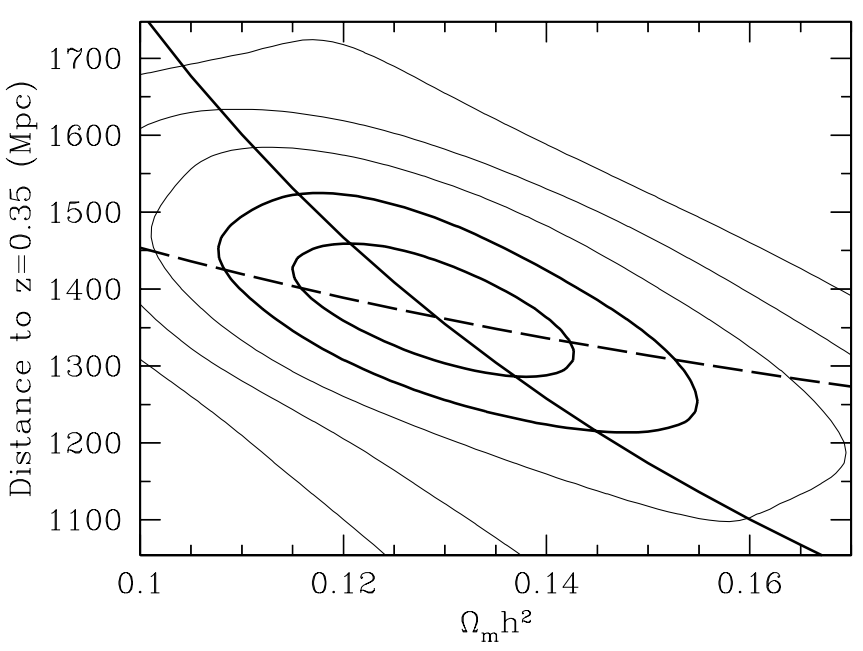

FIG. 7.- Likelihood contours of CDM models as a function of $\Omega_{m} h^{2}$ and $D_{V}(0.35)$. The likelihood has been taken to be proportional to $\exp \left(-\chi^{2} / 2\right)$, and contours corresponding to 1 through $5 \sigma$ for a two-dimensional Gaussian have been plotted. The one-dimensional marginalized values are $\Omega_{m} h^{2}=0.130 \pm$ 0.010 and $D_{V}(0.35)=1370 \pm 64 \mathrm{Mpc}$. We overplot lines depicting the two major degeneracy directions. The solid line is a line of constant $\Omega_{m} h^{2} D_{V}(0.35)$, which would be the degeneracy direction for a pure CDM model. The dashed line is a line of constant sound horizon, holding $\Omega_{b} h^{2}=0.024$. The contours clearly deviate from the pure CDM degeneracy, implying that the peak at $100 h^{-1} \mathrm{Mpc}$ is constraining the fits. [See the electronic edition of the Journal for a color version of this figure.]

sound horizon divided by the distance, which is the apparent location of the acoustic scale. One sees that the long axis of the contours falls in between these two, and the fact that neither direction is degenerate means that both the equality scale and the acoustic scale have been detected. Note that no information from the CMB on $\Omega_{m} h^{2}$ has been used in computing $\chi^{2}$, and so our constraint on $\Omega_{m} h^{2}$ is separate from that from the CMB.

The best-fit pure CDM model has $\chi^{2}=27.8$, which means that it is disfavored by $\Delta \chi^{2}=11.7$ compared to the model with $\Omega_{b} h^{2}=0.024$. Note that we are not marginalizing over the baryon density, so these two parameter spaces have the same number of parameters. The baryon signature is therefore detected at $3.4 \sigma$. As a more stringent version of this, we find that the baryon model is preferred by $\Delta \chi^{2}=8.8(3.0 \sigma)$ even if we only include data points between 60 and $180 \mathrm{~h}^{-1} \mathrm{Mpc}$. Figure 6 also shows $\chi^{2}$ for the pure-CDM model as a function of dilation scale; one sees that the scale constraint on such a model is a factor of 2 worse than the baryonic models. This demonstrates the importance of the acoustic scale in our distance inferences.

As most of our distance leverage is coming from the acoustic scale, the most robust distance measurement we can quote is the ratio of the distance to $z=0.35$ to the distance to $z=1089$ (the redshift of decoupling; Bennett et al. 2003). This marginalizes over the uncertainties in $\Omega_{m} h^{2}$ and would cancel out more exotic errors in the sound horizon, such as from extra relativistic species (Eisenstein \& White 2004). We denote this ratio as

$$
R_{0.35} \equiv \frac{D_{V}(0.35)}{D_{M}(1089)}
$$

Note that the CMB measures a purely transverse distance, while the LRG sample measures the hybrid in equation (2). For the $\Omega_{m}=0.3, \Omega_{\Lambda}=0.7$, and $h=0.7$ cosmology (Tegmark et al. 2004b), $D_{M}(1089)=13700 \mathrm{Mpc}$, with uncertainties due to imperfect measurement of the $\mathrm{CMB}$ angular acoustic scale being negligible at $<1 \%$. We find $R_{0.35}=0.0979 \pm 0.0036$, which is
TABLE 1

Summary of Parameter Constraints from LRGs

\begin{tabular}{|c|c|}
\hline Parameter & Constraint \\
\hline 구라. & $0.130(n / 0.98)^{1.2} \pm 0.011$ \\
\hline$D_{\digamma}(0.35)$ & $1370 \pm 64 \mathrm{Mpc}(4.7 \%)$ \\
\hline$R_{0.35} \equiv D_{V}(0.35) / D_{M}(1089) \ldots \ldots \ldots \ldots$ & $0.0979 \pm 0.0036(3.7 \%)$ \\
\hline$A \equiv D_{V}(0.35)\left(\Omega_{m} H_{0}^{2}\right)^{1 / 2} / 0.35 c \ldots \ldots .$. & $0.469(n / 0.98)^{-0.35} \pm 0.017(3.6 \%)$ \\
\hline
\end{tabular}

Notes.-We assume $\Omega_{b} h^{2}=0.024$ throughout, but variations permitted by $W M A P$ create negligible changes here. We use $n=0.98$, but where variations by 0.1 would create $1 \sigma$ changes, we include an approximate dependence. The quantity $A$ is discussed in $\S 4.5$. All constraints are $1 \sigma$.

a $4 \%$ measurement of the relative distance to $z=0.35$ and $z=$ 1089. Table 1 summarizes our numerical results on these basic measurements and represents our primary results.

To stress that the acoustic scale is responsible for the distance constraint, we repeat our fitting having discarded the two smallest separation bins $\left(10 h^{-1} \mathrm{Mpc}<s<18 h^{-1} \mathrm{Mpc}\right)$ from the correlation function. This is shown in Figure 8. One sees that the constraints on $\Omega_{m} h^{2}$ have degraded (to $0.136 \pm 0.014$ ), but the contours remain well confined along the direction of constant acoustic scale (dashed line). We find a distance ratio of $0.0973 \pm$ 0.0038 , essentially identical to what we found above, with a best $\chi^{2}$ of 13.7 on 15 degrees of freedom.

Varying the spectral tilt, which has an effect similar to including massive neutrinos, is partially degenerate with $\Omega_{m} h^{2}$, but the ratio of the distances is very stable across the plausible range. Repeating the fitting with $n=0.90$ changes the distance ratio $R_{0.35}$ to $0.0986 \pm 0.0041$, a less than $1 \%$ change. The distance itself changes by only $2 \%$ to $D_{V}(0.35)=1344 \pm 70$. The change in the likelihood contours is shown in Figure 9. The best-fit $\Omega_{m} h^{2}$ for $n=0.90$ is $0.143 \pm 0.011$, and so we approximate our $\Omega_{m} h^{2}$ constraint as $0.130(n / 0.98)^{-1.2} \pm 0.011$. While changes of order 0.08 in tilt are marginally allowed with CMB alone, they are strongly disfavored when WMAP is combined with the Ly $\alpha$ forest and galaxy power spectra (Seljak et al. 2005).

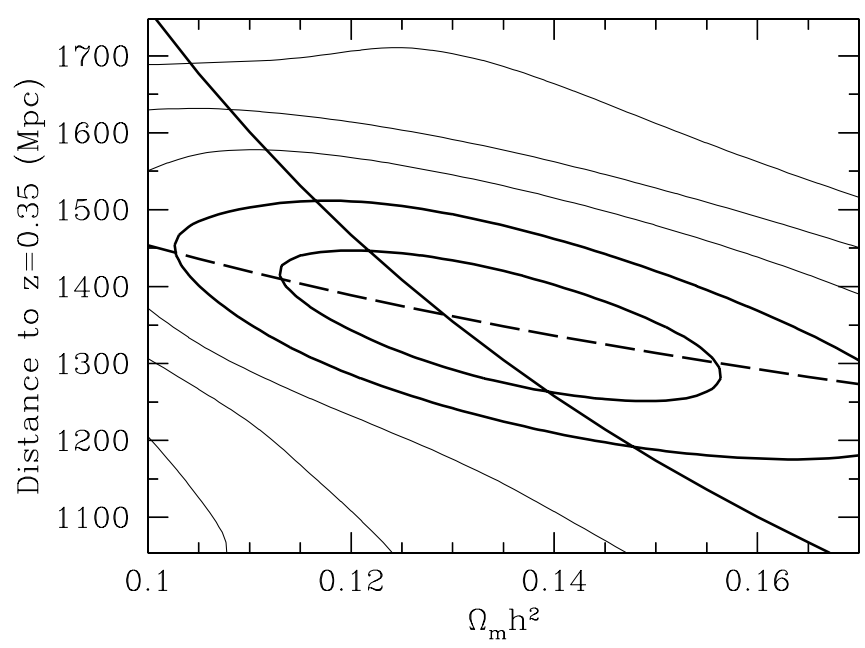

Fig. 8. - Same as Fig. 7, but now with scales below $18 h^{-1}$ Mpc excluded from the $\chi^{2}$ computation. This leaves 18 separation bins and 15 degrees of freedom. The contours are now obviously aligned to the line of constant sound horizon, and the constraints in the $\Omega_{m} h^{2}$ direction are weakened by $40 \%$. As Fig. 3 would suggest, the data at scales below $18 h^{-1}$ Mpc help to constrain $\Omega_{m} h^{2}$, twisting the contours toward the pure CDM degeneracy. Dropping the smaller scales does not affect the constraint on $R_{0.35}$; we find $0.0973 \pm 0.0038$, as compared to $0.0979 \pm 0.0036$ before. [See the electronic edition of the Journal for a color version of this figure.] 


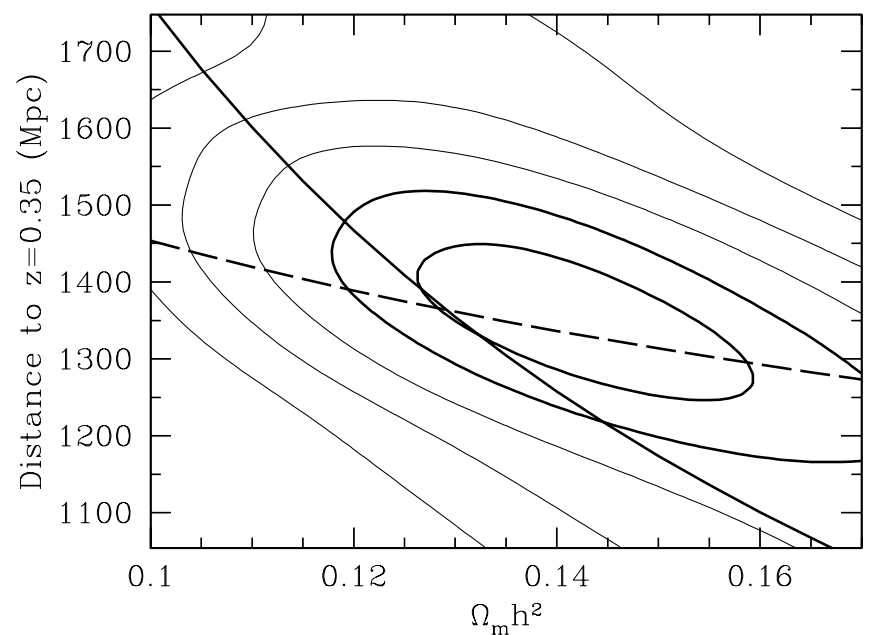

FIG. 9.- Same as Fig. 7, but now with a spectral tilt of $n=0.90$. The best fit has $\chi^{2}=17.8$. The primary effect is a shift to larger $\Omega_{m} h^{2}, 0.143 \pm 0.011$. However, this shift occurs at essentially constant $R_{0.35}$; we find $0.0986 \pm 0.0041$. Again, the acoustic scale robustly determines the distance, even though the spectral tilt biases the measurement of the equality scale. [See the electronic edition of the Journal for a color version of this figure.]

Changing the baryon density to $\Omega_{b} h^{2}=0.030$ still yields a good fit, $\chi^{2}=16.2$, but increases the inferred $\Omega_{m} h^{2}$ to $0.146 \pm$ 0.010 . This is not surprising because higher baryon fractions and lower $\Omega_{m} h^{2}$ both increase the ratio of large- to small-scale power. However, $R_{0.35}$ changes only to $0.0948 \pm 0.0035$. This is a $1 \sigma$ change in $R_{0.35}$, whereas this baryon density change is rejected at $5 \sigma$ by $W M A P$ (Spergel et al. 2003; Tegmark et al. 2004b).

As described in $\S 4.2$, our model correlation functions include two $10 \%$ scale-dependent corrections, the first for nonlinear gravity and the second for scale-dependent bias and redshift distortions. Removing the latter changes $\Omega_{m} h^{2}$ to $0.148 \pm 0.011$, a $2 \sigma$ change from the baseline. We regard ignoring the correction as an extreme alteration. However, even this only moves $R_{0.35}$ to $0.0985 \pm 0.0039$. The best fit itself is worse, as $\chi^{2}$ increases to 19.4. Fitting to the biased, redshift-space correlation function of the $N$-body simulations as compared to the fit to our model for the same cosmology shifts the acoustic scale by only $2 \%$, which is $0.5 \sigma$. We therefore conclude that systematic errors in the theoretical modeling are subdominant to our statistical errors.

The contour plots are based on the covariance matrix derived from the mock catalogs. To validate this, we consider the scatter in the best-fit model parameters among the 10 jackknife subsamples (see discussion in $\S 3.3$ ). The jackknifed error in $\Omega_{m} h^{2}$ is 0.011 , that in $D_{V}(0.35)$ is $4.6 \%$, and that in the distance ratio $R_{0.35}$ is $3.2 \%$. These values are close to those found from the $\chi^{2}$-based likelihood function $(0.011,4.8 \%$, and $3.7 \%$, respectively). This justifies the likelihood contours derived from the covariance matrix. It also demonstrates that our results are not being driven by one unusual region of the survey.

\subsection{Constraints on Dark Energy and Spatial Curvature}

For fixed values of $\Omega_{m} h^{2}$ and $\Omega_{b} h^{2}$, the angular scale of the $\mathrm{CMB}$ acoustic peaks constrains the angular diameter distance to $z=1089$ to very high accuracy. If one considers only a simple parameter space of flat cosmologies with a cosmological constant, then this distance depends only on one parameter, say $\Omega_{m}$ or $\Omega_{\Lambda}$ (the two must sum to unity, and $H_{0}$ is then fixed by the value of $\Omega_{m} h^{2}$ ), and so the distance measurement constrains $\Omega_{m}$, $\Omega_{\Lambda}$, and $H_{0}$ to high precision. If one generalizes to larger parameter spaces, e.g., adding an unknown dark energy equation of state $w(z)$ (Turner \& White 1997; Caldwell et al. 1998) or a nonzero curvature, then a parameter degeneracy opens in the CMB (e.g., Eisenstein et al. 1998b; Efstathiou \& Bond 1999). The acoustic scale still provides one high-quality constraint in this higher dimensional space, but the remaining directions are constrained only poorly by gravitational lensing of the CMB (e.g., Seljak 1996; Stompor \& Efstathiou 1999) and the integrated Sachs-Wolfe effect on small and large angular scales, respectively.

With our measurement of the acoustic scale at $z=0.35$, we can add another high-quality constraint, thereby yielding good measurements on a two-dimensional space (e.g., a constant $w \neq$ -1 or a nonzero curvature). A more general $w(z)$ model would of course require additional input data, e.g., large-scale structure at another redshift, supernovae distance measurements, or a Hubble constant measurement.

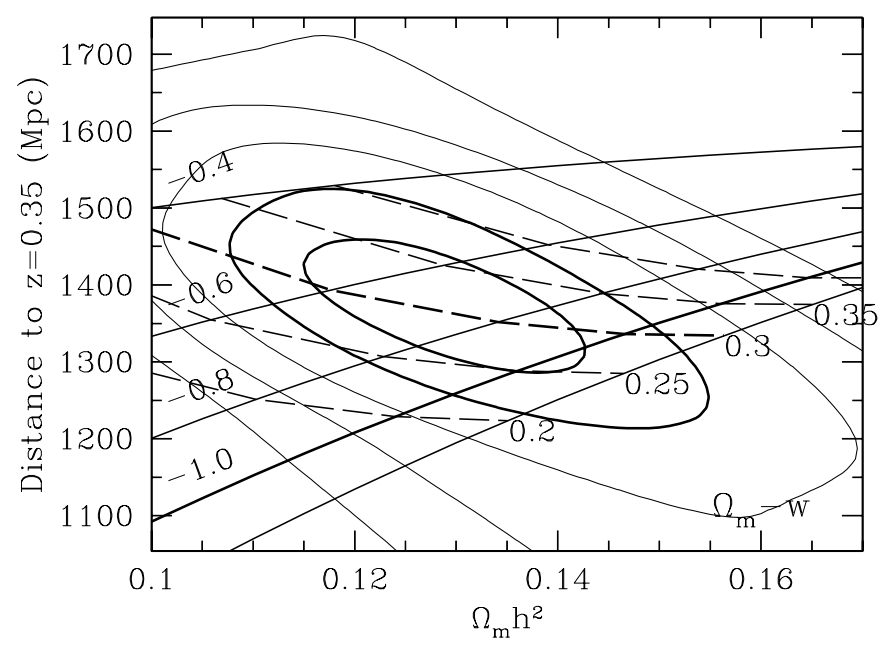

FIG. 10a

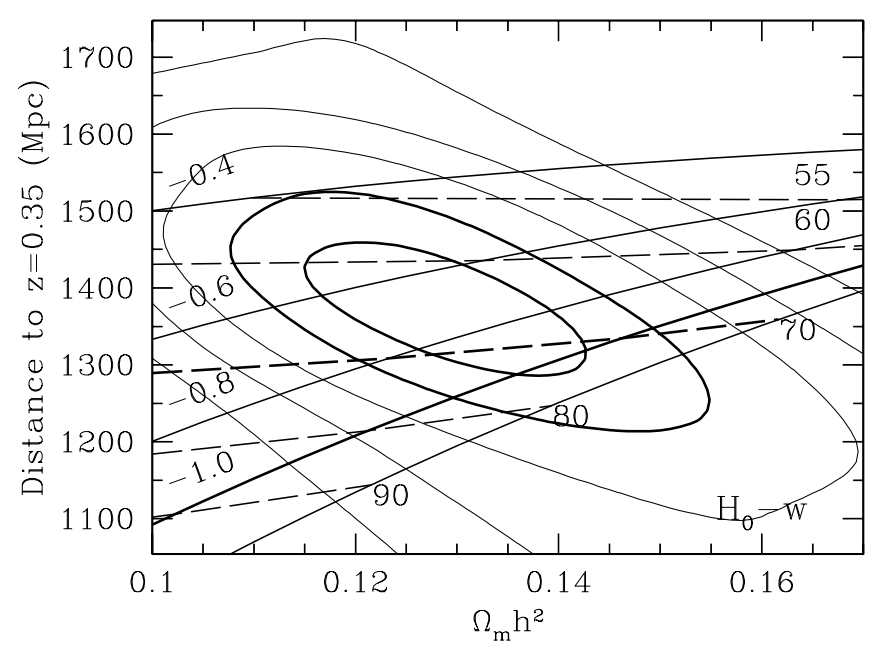

FIG. $10 b$

FIG. 10.- (a) Same as Fig. 7, but overplotted with model predictions from constant- $w$ flat models. For a given value of $\Omega_{m} h^{2}$ and $w$, the angular scale of the CMB

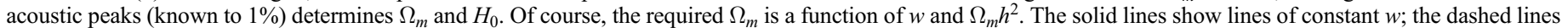

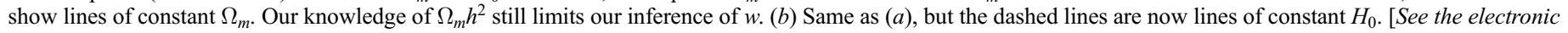
edition of the Journal for a color version of this figure.] 


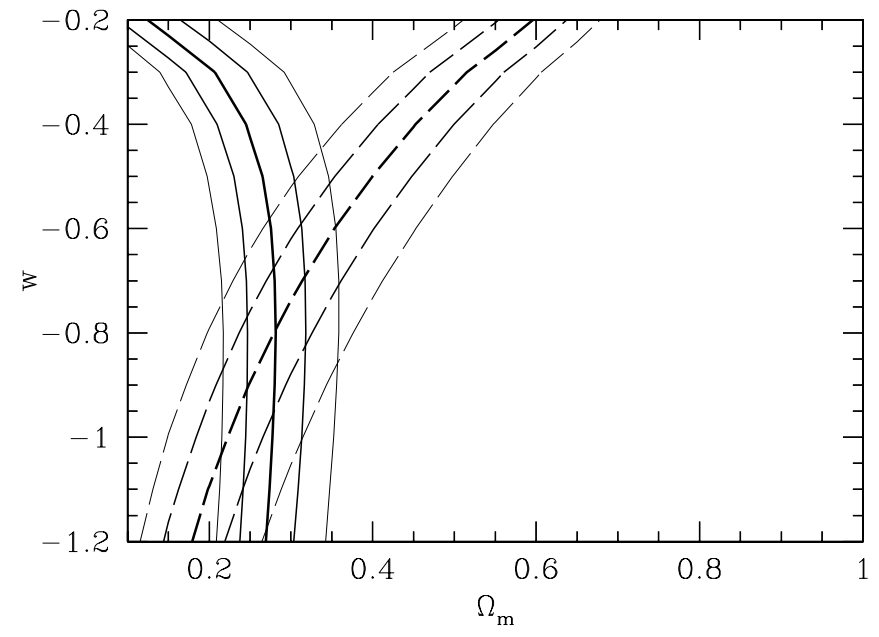

FIG. 11.-Contours in the space of $\Omega_{m}$ and $w$. The solid contours show the lines of constant $R_{0.35}$ (from 0.090 to 0.106 , with the central value of 0.098 ). The dashed contours show the contours of constant $\Omega_{m} h^{2}$, using the angular scale of the CMB acoustic peaks to set $H_{0}$ at each $\left(\Omega_{m}, w\right)$ pair. The values of $\Omega_{m} h^{2}$ range from 0.11 to 0.15 , which is the -2 to $2 \sigma$ range from Fig. 7. Uncertainties in the value of $\Omega_{m} h^{2}$ significantly impact our constraints on $w$. [See the electronic edition of the Journal for a color version of this figure.]

For the simple space of flat cosmologies with a constant $w \neq$ -1 , at each value of $\Omega_{m} h^{2}$ and $w$ we can find the value of $\Omega_{m}$ (or $H_{0}$ ) that yields the correct angular scale of the acoustic peak and then use this value to predict $D_{V}(z=0.35)$. In Figure $10 a$ we overlay our constraints with the grid of $\Omega_{m}$ and $w$ inferred in this way. One sees that $\Omega_{m}$ is well constrained but that $w$ is not. The reason for the latter is that $\Omega_{m} h^{2}$ is not yet known well enough. This is illustrated in Figure 11 . Were $\Omega_{m} h^{2}$ known to $1 \%$, as is expected from the Planck ${ }^{30}$ mission, then our constraints on $w$ would actually be better than 0.1 . Figure $10 b$ shows the constraints with a grid of $H_{0}$ and $w$ overlaid.

Tegmark et al. (2004b) used a Markov chain analysis of the WMAP data combined with the SDSS main sample galaxy power spectrum to constrain cosmological parameters. They found $\Omega_{m} h^{2}=0.145 \pm 0.014, w=-0.92 \pm 0.30, \Omega_{m}=$ $0.329 \pm 0.074$, and $h=0.68 \pm 0.10$ (varying also $n, \Omega_{b}$, the optical depth $\tau$, and a linear bias). Here we use the mean and standard deviation rather than the asymmetric quantiles in Tegmark et al. (2004b), and we use a prior of $\tau<0.3$. Adding the

30 See http://www.rssd.esa.int/index.php?project=PLANCK.
LRG measurement of $R_{0.35}$ and the constraint that $\Omega_{m} h^{2}=$ $0.130(n / 0.98)^{-1.2} \pm 0.011$, we find $\Omega_{m} h^{2}=0.135 \pm 0.008, w=$ $-0.80 \pm 0.18, \Omega_{m}=0.326 \pm 0.037$, and $h=0.648 \pm 0.045$. We are ignoring the small overlap in survey region between SDSS main sample and the LRG sample. The improvements in $w$ arise primarily from the constraint on $\Omega_{m} h^{2}$, while the improvements in $\Omega_{m}$ come more from the measurement of $R_{0.35}$. Table 2 summarizes all of the numerical results from this section.

It is important to remember that constant- $w$ models are not necessarily good representations of physical models of dynamical dark energy and that forcing this parameterization can lead to bias (Maor et al. 2002; Bassett et al. 2004). We offer the previous analysis as a means to compare to the literature, but we prefer our actual distance measurements in Table 1 as a modelindependent set of constraints.

We next turn to the space of models with two well-specified ingredients, namely a cosmological constant (i.e., $w=-1$ ) and nonzero spatial curvature. The results are in Figure 12. Unlike the constraints on $w \neq-1$, the constraints on the spatial curvature are excellent, of order $1 \%$. This is because the distance to $z=1089$ is extremely sensitive to spatial curvature, such that we get excellent performance by supplying a calibration of the distance scale (e.g., $H_{0}$ with a touch of $\Omega_{m}$ ) at low redshift. Of course, this is in accord with the conventional wisdom that the $\mathrm{CMB}$ constrains the universe to be nearly flat, but our result represents a significant tightening of the angular diameter distance degeneracy.

Using the Tegmark et al. (2004b) Markov chain results for a $w=-1$ cosmology with spatial curvature, the SDSS main sample $P(k)$ plus $W M A P$ produces $\Omega_{m} h^{2}=0.134 \pm 0.012, \Omega_{K}=$ $-0.045 \pm 0.032, \Omega_{m}=0.43 \pm 0.096$, and $h=0.57 \pm 0.08$. Adding the $R_{0.35}$ constraint from the SDSS LRG results, we find $\Omega_{m} h^{2}=0.142 \pm 0.011, \Omega_{K}=-0.006 \pm 0.011, \Omega_{m}=0.309 \pm$ 0.086 , and $h=0.679 \pm 0.033$. Adding the further information on $\Omega_{m} h^{2}$ drops the values to $\Omega_{m} h^{2}=0.136 \pm 0.008, \Omega_{K}=$ $-0.010 \pm 0.009, \Omega_{m}=0.306 \pm 0.027$, and $h=0.669 \pm 0.028$. Hence, the essential improvement comes from the measurement of $R_{0.35}$; with it, we find that the universe is flat to $1 \%$ accuracy, assuming that $w=-1$.

If we require a flat cosmology with $w=-1$, then the Markov chain analysis from the WMAP, main, and LRG data together yields $\Omega_{m} h^{2}=0.142 \pm 0.005, \Omega_{m}=0.296 \pm 0.025$, and $h=$ $0.692 \pm 0.021$. The $W M A P$ data alone is not strongly degenerate in this parameter space (Spergel et al. 2003), although the galaxy data do tighten the constraints by roughly a factor of 3 . One could also read $\Omega_{m}$ and $h$ directly from the "flat" line in Figure 12. This

TABLE 2

Joint Constraints on Cosmological Parameters including CMB Data

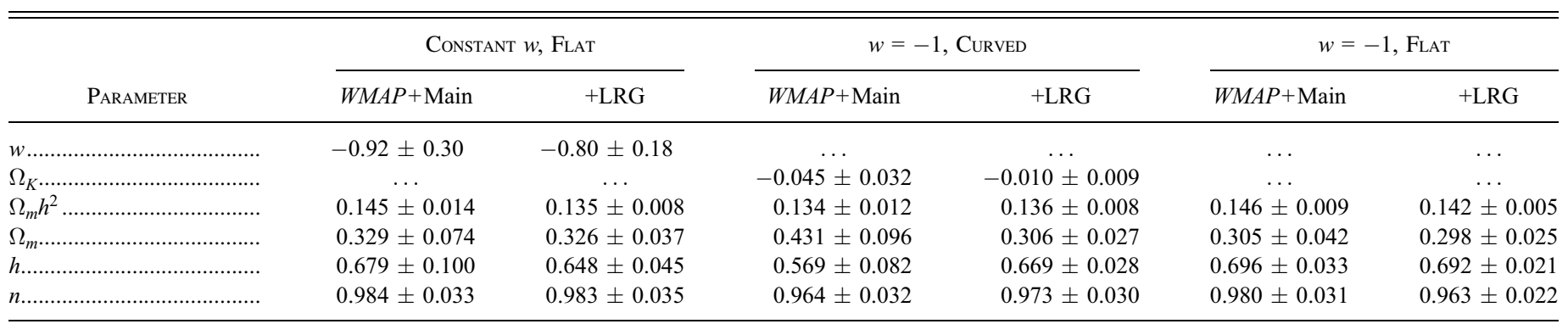

Notes.-Constraints on cosmological parameters from the Markov chain analysis. The first two data columns are for spatially flat models with constant $w$, while the next two are for $w=-1$ models with spatial curvature. In each case, the other parameters are $\Omega_{m} h^{2}, \Omega_{b} h^{2}, n_{s}, h$, and the optical depth $\tau$ (which we have required to be less than 0.3 ). A negative $\Omega_{K}$ means a spherical geometry. The mean values are listed with the $1 \sigma$ errors. The first column in each set gives the constraints from Tegmark et al. (2004b) from combining $W M A P$ and the SDSS main sample. The second column adds our LRG constraints: $R_{0.35}=0.0979 \pm 0.036$ and $\Omega_{m} h^{2}=0.130(n / 0.98)^{-1.2} \pm 0.011$. In all cases, $\Omega_{b} h^{2}$ is constrained by the CMB to an accuracy well below where we would need to include variations in the LRG analysis. 


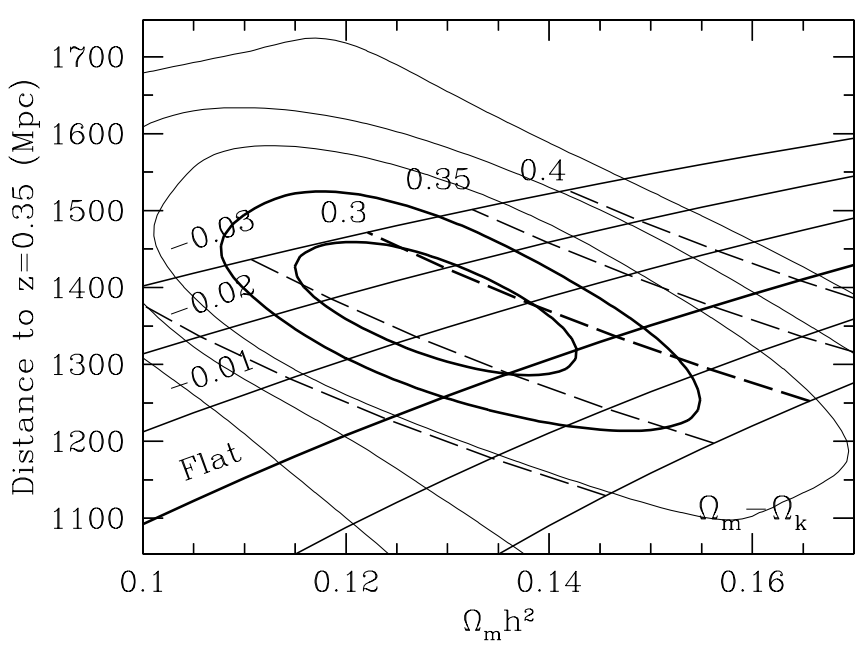

Fig. $12 a$

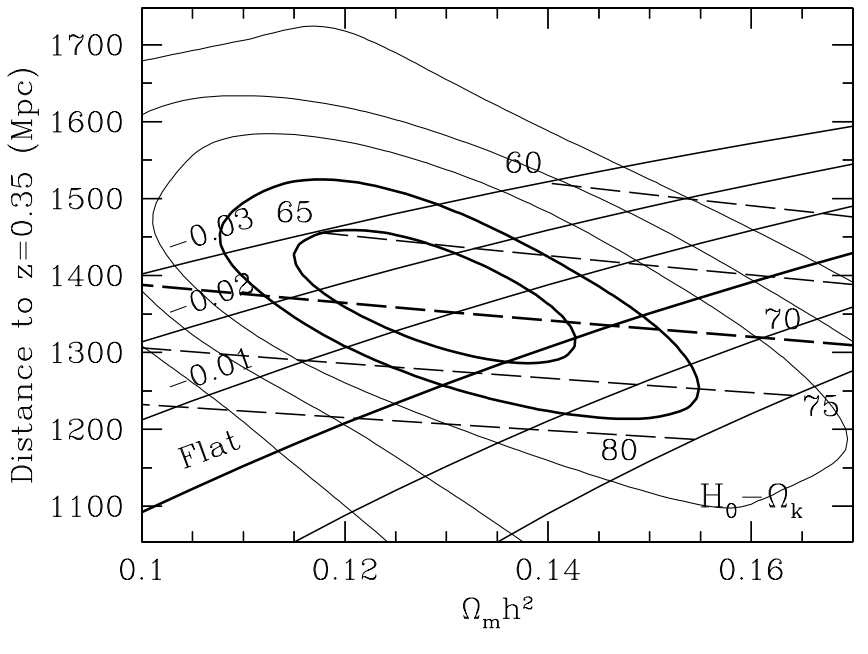

FIG. $12 b$

FIG. 12.-(a) Same as Fig. 10, but now overplotted with models of $w=-1$ but nonzero curvature. The solid lines are lines of constant curvature, running from -0.03 (closed) to +0.02 (open). The dashed lines are lines of constant $\Omega_{m}$, from 0.20 to 0.40 . The constraints on the curvature of the universe are superb. This happens because we are combining the low-redshift distance scale with the distance to $z=1089$, by which point even tiny amounts of curvature make a big difference. $(b)$ Same as $(a)$, but the dashed lines are now lines of constant $H_{0}$. [See the electronic edition of the Journal for a color version of this figure.]

gives $\Omega_{m}=0.271 \pm 0.022$ and $h=0.723 \pm 0.017$ at a fixed spectral tilt $n=0.98$. The difference occurs because the WMAP data and the SDSS main sample pull the value of $\Omega_{m} h^{2}$ higher and because the best-fit tilt in the Markov chain is below $n=0.98$.

\subsection{Low-Redshift Cosmological Constraints}

In more general dark energy models, the $R_{0.35}$ measurement will not measure $\Omega_{K}$ or $w(z)$ by itself. However, the redshift of the LRG sample is low enough that we can get interesting constraints focusing on the path from $z=0$ to $z=0.35$ rather than $z=0.35$ to $z=1089$. We note that the combination $D_{V}(0.35)\left(\Omega_{m} h^{2}\right)^{1 / 2}$ has no dependence on the Hubble constant $H_{0}$, since $D_{V}(0.35)$ is proportional to $H_{0}^{-1}$ [times a function of all the $\Omega$ 's and $w(z)$ ]. Fortuitously, this combination is well constrained by our data, as these contours lie along the long axis of our constraint region. We measure

$$
A \equiv D_{V}(0.35) \frac{\sqrt{\Omega_{m} H_{0}^{2}}}{0.35 c}=0.469 \pm 0.017(3.6 \%)
$$

This value is robust against changes in the minimum scale of data used in the fit $\left(0.471 \pm 0.021\right.$ for $\left.r>18 h^{-1} \mathrm{Mpc}\right)$, the spectral tilt $(0.483 \pm 0.018$ for $n=0.90)$, and the baryon density $\left(0.468 \pm 0.017\right.$ for $\left.\Omega_{b} h^{2}=0.030\right)$. As $A$ is independent of a dark energy model, we include its value in Table 1 .

If the LRG redshift were closer to 0 , then $A$ would simply be $\left(\Omega_{m}\right)^{1 / 2}$. At $z=0.35, A$ depends weakly on $\Omega_{K}$ and on $w(z)$ over the range $0<z<0.35$. In detail, for a flat universe and constant $w$, which we denote as $w_{0}$ given the low redshift, we have

$$
A=\sqrt{\Omega_{m}} E\left(z_{1}\right)^{-1 / 3}\left[\frac{1}{z_{1}} \int_{0}^{z_{1}} \frac{d z}{E(z)}\right]^{2 / 3}
$$

where $E(z)=H(z) / H_{0}=\left[\Omega_{m}(1+z)^{3}+\Omega_{\Lambda}(1+z)^{3+3 w_{0}}\right]^{1 / 2}$ and $z_{1}=0.35$. The generalization to curved spaces is straightforward. While treating $w$ as a constant for all times may be a poor model (e.g., Weller \& Albrecht 2002; Bassett et al. 2004; Maor et al. 2002; Virey et al. 2004), it is a reasonable approximation for so short an interval. In detail, $w_{0}$ is not the value at $z=0$ but rather some average out to $z=0.35$.
We therefore linearize the expression for $A$ in $\Omega_{m}, \Omega_{K}$, and $w_{0}$ to find

$$
\Omega_{m}=0.273+0.123\left(1+w_{0}\right)+0.137 \Omega_{K} \pm 0.025 .
$$

This result relies on the acoustic length scale being predicted correctly at $z \sim 1000$ from the CMB measurement of $\Omega_{b} h^{2}$ and the LRG measurement of $\Omega_{m} h^{2}$, but it is independent of the angular acoustic scale in the CMB and hence makes no assumption about $w(z)$ at $z>0.35$. It will depend slightly on unmarginalized parameters such as the spectral tilt, the neutrino mass, or other manners of altering the LRG value of $\Omega_{m} h^{2}$. As we demonstrated in $\S 4.4$, the CMB acoustic scale is very sensitive to $\Omega_{K}$; invoking a large $\left|\Omega_{K}\right|$ in equation (6) would require large contortions in $w(z)$ to maintain the angular location of the acoustic peaks. The error in equation (6) is consistent with the error on $\Omega_{m}$ in the constant- $w$ Markov chain because the uncertainties in $w_{0}$ increase the allowed range of $\Omega_{m}$.

\section{CONCLUSIONS}

We have presented the large-scale correlation function from the SDSS Luminous Red Galaxy sample. This is the largest effective volume yet surveyed by a factor of $\sim 4$ at small wavenumber. We find clear evidence $(3.4 \sigma)$ for the acoustic peak at $100 h^{-1} \mathrm{Mpc}$ scale. The scale and amplitude of this peak are in excellent agreement with the prediction from the $\Lambda \mathrm{CDM}$ interpretations of CMB data, such as from WMAP. Moreover, the broadband shape of the rest of the correlation function gives a measurement of the matter density $\Omega_{m} h^{2}$ that matches the CMB findings. Tables 1 and 2 and equation (6) list our quantitative results.

Before reviewing the quantitative conclusions, we focus on the more fundamental ones. The imprint of the acoustic oscillations on the low-redshift clustering of matter is a generic prediction of CDM cosmological theory (Peebles \& Yu 1970; Bond \& Efstathiou 1984; Holtzmann 1989; Hu \& Sugiyama 1996). Our detection confirms two aspects of the theory: first, that the oscillations occur at $z \gtrsim 1000$, and second that they survive the intervening time to be detected at low redshift. The small amplitude of the features requires that there exist matter at $z \sim 1000$ that does not interact with the photon-baryon fluid, i.e., dark 
matter. Fully baryonic models or those with extra interacting matter produce much stronger acoustic signatures that would have to be erased by some exotic later process to match low-redshift observations.

In CDM models, large-scale fluctuations have grown since $z \sim 1000$ by gravitational instability. In particular, perturbation theory predicts that small perturbations grow in a manner that leaves the Fourier modes of the density field uncoupled. This in turn protects the narrow features such as the acoustic oscillations as they grow. Nonlinear gravitational perturbation theories generically predict mode coupling that would wash out the acoustic signature (e.g., Fry 1984; Goroff et al. 1986; Jain \& Bertschinger 1994).

Hence, the detection of low-redshift acoustic oscillations is a stark confirmation of the CDM theory for the growth of cosmological structure and the link between the CMB anisotropies and the matter perturbations. While the agreement between recent results on the broadband shape of the matter power spectrum (Efstathiou et al. 2002; Tegmark et al. 2004a; McDonald et al. 2004), the galaxy three-point correlation function (e.g., Feldman et al. 2001), and the inferences from the CMB (e.g., Spergel et al. 2003) was certainly compelling on this point, we regard the acoustic signature as a smoking gun, as its narrowness in real space would be difficult to mimic in alternative models of structure formation. This detection confirms the applicability of linear cosmological perturbation theory on large scales and across a factor of 800 in cosmic expansion.

The narrowness of the acoustic peak in real space offers an opportunity to measure distances to higher redshifts (Eisenstein et al. 1998b; Eisenstein 2002; Blake \& Glazebrook 2003). It is worth noting that this is a circumstance where a given improvement in signal-to-noise ratio in the clustering statistic makes a superlinear improvement in the distance constraint. One can draw an analogy to the determination of the redshift of a galaxy with an emission line. A factor of 2 in signal-to-noise ratio can make the difference between detecting the line, and hence constraining the redshift to very high precision, and not detecting it and having to rely on the spectral shape for a low-precision photometric redshift. In the case of large-scale clustering, the acoustic scale is not as narrow and so the improvement is less dramatic, but we clearly benefit at the factor of 2 level from using the acoustic scale rather than the broadband shape of the correlation function (i.e., the equality scale).

In the LRG sample, we measure the acoustic scale to just better than $4 \%$ precision $(1 \sigma)$. Comparing this scale to the angular scale of the CMB anisotropies gives the distance ratio $R_{0.35}=$ $D_{V}(0.35) / D_{M}(1089)=0.0979 \pm 0.0036$, where $D_{V}(0.35)$ is defined in equation (2). This distance ratio is robust against changes in the broadband clustering signal such as via the spectral tilt and against variations in our analysis. It is also robust against certain kinds of exotica, such as adding additional relativistic energy to the universe (Eisenstein \& White 2004). It does rely on the wellunderstood linear perturbation theory of the recombination epoch to relate the perturbations in the photons to those in the matter. Given this theory, we have measured the relative distance between two radically different redshifts using a purely geometric method and the same physical mechanism.

This distance ratio is consistent with the familiar cosmological constant cosmology. It is grossly inconsistent with the Einsteinde Sitter $\left(\Omega_{m}=1\right)$ model, which predicts $R_{0.35}=0.133$ (nominally $10 \sigma$ ). A model lacking dark energy would require $\Omega_{m}=$ 0.70 with $\Omega_{K}=0.30$ to match the distance ratio. This would require $h=0.90$ and $\Omega_{m} h^{2}=0.57$ to match the CMB peak location, implying an age of 8 Gyr. This is in complete disagreement with the observed shape of the CMB anisotropy spectrum, the galaxy correlation function (including these LRG data), the cluster baryon fraction (White et al. 1993), the observed value of $H_{0}$ (Freedman et al. 2001), and the age of old stars (Krauss \& Chaboyer 2003 and references therein), as well as other cosmological measurements. Hence, our measurement provides geometric evidence for dark energy.

The size of the acoustic scale is predicted by very simple physics, namely the comoving distance that a sound wave can travel between the generation of the perturbations and the epoch of recombination. In the standard cosmological model, this depends only on the matter density $\Omega_{m} h^{2}$ and baryon density $\Omega_{b} h^{2}$. The uncertainties on $\Omega_{b} h^{2}$ from CMB and big bang nucleosynthesis are small, contributing $<2 \%$ to the error on the acoustic scale. However, current uncertainties in $\Omega_{m} h^{2}$ are large enough that we need to track the covariance of $\Omega_{m} h^{2}$ with our distance inferences.

Because the acoustic scale is detected, the LRG data alone constrain the equality scale and matter density; i.e., we measure $\Omega_{m} h^{2}$ rather than the more familiar $\Gamma=\Omega_{m} h$. With our baseline method and $\Omega_{b} h^{2}=0.024$, we find $\Omega_{m} h^{2}=0.130(n / 0.98)^{1.2} \pm$ 0.010 . This precision is similar to that from current CMB measurements (Spergel et al. 2003). Importantly, the LRG value agrees with the CMB value and with the inference from the clustering of the lower redshift SDSS main galaxy sample (Tegmark et al. 2004a), a remarkable cosmological consistency test. Because the formal precision of the LRGs is as good as the other measurements, we choose to use only the LRGs in our fitting. Adding the WMAP information on $\Omega_{m} h^{2}$ as an external prior would improve the quantitative constraints only slightly, and so we leave it as a cross-check. We expect our knowledge of $\Omega_{m} h^{2}$ to improve rapidly in the coming years both from the CMB, with additional $W M A P$ data and smaller angle ground-based observations, and from large-scale structure, e.g., with improved modeling of scales below $10 \mathrm{~h}^{-1} \mathrm{Mpc}$ and the continued data collection for the SDSS LRG sample.

Using the LRG value for $\Omega_{m} h^{2}$, we find the distance to $z=$ 0.35 to be $D_{V}(0.35)=1370 \pm 64 \mathrm{Mpc}$, a 5\% measurement. Were this at $z \sim 0$, we would have a measurement of $H_{0}$ and $\Omega_{m}$, but at $z=0.35$ dark energy and curvature do matter. The combination $D_{V}(0.35)\left(\Omega_{m} H_{0}^{2}\right)^{1 / 2} / 0.35 c$ is measured to $4 \%$ precision and is independent of $H_{0}$. From this, we infer $\Omega_{m}=0.273+$ $0.123\left(1+w_{0}\right)+0.137 \Omega_{K} \pm 0.025$, where this $w_{0}$ is the effective value in the range $0<z<0.35$.

Combining with the $\mathrm{CMB}$ acoustic scale, we put constraints on more restricted models, either constant $w$ or $w=-1$ plus curvature. We find that our $w$ leverage is roughly 0.2 . Improvements in knowledge of $\Omega_{m} h^{2}$ will help significantly. Our leverage on spatial curvature is exquisite: we measure $\Omega_{K}=$ $-0.010 \pm 0.009$. Of course, this is a manifestation of the wellknown sensitivity of the CMB to spatial curvature, but we are breaking the angular diameter distance degeneracy with the best precision to date and with a distance ratio that relies on the same physics as the CMB.

It is important to note that because the low-redshift acoustic oscillation method measures distances that can be quantitatively compared to those from the $\mathrm{CMB}$, the method retains sensitivity to phenomena that have more effect at higher redshift, such as curvature. Relative distance methods, such as supernovae, can only constrain the Hubble relation out to the maximum redshift of the sample, but absolute methods probe both above and below that redshift by using both $z=0$ and the CMB as comparison points.

It is interesting to compare the provenance of the absolute distance measurements offered by the acoustic scale to those of the classical measurements of $H_{0}$ (e.g., Freedman et al. 2001). 
Once established at $z>1000$, the acoustic scale can be used at low redshift as a standard ruler on a footing equal to that of any other. The issue of course is that the early universe $\left(10^{3}<z<\right.$ $10^{5}$ ) is a remote place to calibrate one's ruler. There are assumptions about the relativistic energy density, the adiabatic nature of the perturbations, the early generation $\left(z \gtrsim 10^{5}\right)$ of the perturbations, and the absence of particle decays at $z \lesssim 10^{5}$ (Eisenstein $\&$ White 2004). Many possible alterations create glaring deviations in the CMB anisotropies (e.g., Moodley et al. 2004). Others, such as small alterations to the relativistic density, are more subtle, at least with present data. Our sense is that altering the acoustic scale so as to misestimate $H_{0}$ will require some interesting piece of new fundamental physics. The future of direct $H_{0}$ studies may be as a probe of high-redshift particle physics!

This detection of the acoustic peak at low redshift dramatically confirms several basic assumptions of cosmological structure formation theory, but it also points the way to a new application of large-scale structure surveys for the study of dark energy. Survey volumes of order $1 h^{-3} \mathrm{Gpc}^{3}$ offer a reliable standard ruler, whose measurement across a range of redshifts can determine $H(z)$ and $D_{A}(z)$ robustly to percent-level accuracy (Blake \& Glazebrook 2003; Hu \& Haiman 2003; Seo \& Eisenstein 2003). Indeed, the available precision improves at higher redshift because the acoustic peak is less broadened by nonlinear structure formation. The observational challenge is to execute these large, wide-field surveys at $z>0.5$.
We thank Scott Dodelson, Sebastian Jester, and Ravi Sheth for useful comments. D. J. E. and D. W. H. thank the Laboratoire de Physique Théorique, Université de Paris XI, for hospitality. D. J. E., I. Z., and H. S. are supported by grant AST 00-98577 and AST 04-07200 from the National Science Foundation. D. J. E. is further supported by an Alfred P. Sloan Research Fellowship. D. W. H. and M. R. B. were supported by NSF PHY 01-01738 and NASA NAG5-11669. Our PTHALOS mock catalogs were created on the NYU Beowulf cluster supported by NSF grant PHY 01-16590.

Funding for the creation and distribution of the SDSS Archive has been provided by the Alfred P. Sloan Foundation, the Participating Institutions, the National Aeronautics and Space Administration, the National Science Foundation, the US Department of Energy, the Japanese Monbukagakusho, and the Max Planck Society. The SDSS Web site is http://www.sdss.org.

The SDSS is managed by the Astrophysical Research Consortium (ARC) for the Participating Institutions. The Participating Institutions are the University of Chicago, Fermilab, the Institute for Advanced Study, the Japan Participation Group, the Johns Hopkins University, the Korean Scientist Group, Los Alamos National Laboratory, the Max-Planck-Institute for Astronomy (MPIA), the Max-Planck-Institute for Astrophysics (MPA), New Mexico State University, the University of Pittsburgh, the University of Portsmouth, Princeton University, the United States Naval Observatory, and the University of Washington.
Abazajian, K., et al. 2003, AJ, 126, 2081 $2004, \mathrm{AJ}, 128,502$

Alcock, C., \& Paczyński, B. 1979, Nature, 281, 358

Amendola, L., Quercellini, C., \& Giallongo, E., 2005, MNRAS, 357, 429

Bardeen, J. M., Bond, J. R., Kaiser, N., \& Szalay, A. S. 1986, ApJ, 304, 15

Bashinsky, S., \& Bertschinger, E. 2001, Phys. Rev. Lett., 87, 081301

- 2002, Phys. Rev. D, 65, 123008

Bassett, B. A., Corasaniti, P. S., \& Kunz, M. 2004, ApJ, 617, L1

Bennett, C., et al. 2003, ApJS, 148, 1

Benoît, A., et al. 2003, A\&A, 399, L19

Berlind, A. A., et al. 2003, ApJ, 593, 1

Blake, C., \& Glazebrook, K. 2003, ApJ, 594, 665

Blanton, M. R., Lupton, R. H., Maley, F. M., Young, N., Zehavi, I., \& Loveday, J. 2003, AJ, 125, 2276

Blanton, M. R., et al. 2005, AJ, 129, 2562

Bond, J. R., \& Efstathiou, G. 1984, ApJ, 285, L45 1987, MNRAS, 226, 655

Bond, J. R., \& Szalay, A. 1983, ApJ, 274, 443

Broadhurst, T. J., Ellis, R. S., Koo, D. C., \& Szalay, A. S. 1990, Nature, 343, 726

Burles, S., Nollett, K. M., \& Turner, M. S. 2001, Phys. Rev. D, 63, 063512

Caldwell, R. R., Dave, R., \& Steinhardt, P. J. 1998, Phys. Rev. Lett., 80, 1582

Coc, A., Vangioni-Flam, E., Descouvemont, P., Adahchour, A., \& Angulo, C.

2004, ApJ, 600, 544

Coles, P. 1993, MNRAS, 262, 1065

Colless, M., et al. 2001, MNRAS, 328, 1039

2003, preprint (astro-ph/0306581)

Cooray, A., \& Sheth, R. 2002, Phys. Rep., 372, 1

Croom, S. M., Smith, R. J., Boyle, B. J., Shanks, T., Miller, L., Outram, P. J., \&

Loaring, N. S. 2004, MNRAS, 349, 1397

Croom, S. M., et al. 2005, MNRAS, 356, 415

de Bernardis, P., et al. 2000, Nature, 404, 955

Dolney, D., Jain, B., \& Takada, M. 2004, preprint (astro-ph/0409445)

Efstathiou, G., \& Bond, J. R. 1999, MNRAS, 304, 75

Efstathiou, G., et al. 2002, MNRAS, 330, L29

Einasto, J., et al. 1997, Nature, 385, 139

Eisenstein, D. J. 2002, in ASP Conf. Ser. 280, Next Generation Wide-Field Multi-Object Spectroscopy, ed. M. J. I. Brown \& A. Dey (San Francisco: ASP), 35

Eisenstein, D. J., \& Hu, W. 1998, ApJ, 496, 605

Eisenstein, D. J., Hu, W., Silk, J., \& Szalay, A. S. 1998a, ApJ, 494, L1

Eisenstein, D. J., Hu, W., \& Tegmark, M. 1998b, ApJ, 504, L57

Eisenstein, D. J., \& White, M. 2004, Phys. Rev. D, 70, 103523
REFERENCES

Eisenstein, D. J., et al. 2001, AJ, 122, 2267

2003, ApJ, 585, 694

2005, ApJ, 619, 178

Feldman, H. A., Frieman, J. A., Fry, J. N., \& Scoccimarro, R. 2001, Phys. Rev. Lett., 86, 1434

Feldman, H. A., Kaiser, N., \& Peacock, J. A. 1994, ApJ, 426, 23

Finkbeiner, D., et al. 2004, AJ, 128, 2577

Freedman, W. L., et al. 2001, ApJ, 553, 47

Fry, J. N. 1984, ApJ, 279, 499

Fry, J. N., \& Gaztanaga, E. 1993, ApJ, 413, 447

Fukugita, M., Ichikawa, T., Gunn, J. E., Doi, M., Shimasaku, K., \& Schneider, D. P. 1996, AJ, 111, 1748

Goldberg, D. M., \& Strauss, M. 1998, ApJ, 495, 29

Goroff, M. H., Grinstein, B., Rey, S.-J., \& Wise, M. B. 1986, ApJ, 311, 6

Gunn, J. E., et al. 1998, AJ, 116, 3040

Halverson, N. W., et al. 2002, ApJ, 568, 38

Hamilton, A. J. S. 1998, in The Evolving Universe, ed. D. Hamilton (Dordrecht: Kluwer), 185

Hanany, S., et al. 2000, ApJ, 545, L5

Hawkins, E., et al. 2003, MNRAS, 346, 78

Hogg, D. W., Eisenstein, D. J., Blanton, M. R., Bahcall, N. A., Brinkmann, J., Gunn, J. E., \& Schneider, D. P. 2005, ApJ, 624, 54

Hogg, D. W., Finkbeiner, D. P., Schlegel, D. J., \& Gunn, J. E. 2001, AJ, 122, 2129

Hogg, D. W., et al. 2003, ApJ, 585, L5

Holtzmann, J. A. 1989, ApJS, 71, 1

Hu, W. 2005, in ASP Conf. Ser. 339, Observing Dark Energy, ed. S. C. Wolff \&

T. R. Lauer (San Francisco: ASP), 215

Hu, W., \& Dodelson, S. 2002, ARA\&A, 40, 171

Hu, W., \& Haiman, Z. 2003, Phys. Rev. D, 68, 063004

Hu, W., Spergel, D. N., \& White, M. 1997a, Phys. Rev. D, 55, 3288

Hu, W., \& Sugiyama, N. 1996, ApJ, 471, 542

Hu, W., Sugiyama, N., \& Silk, J. 1997b, Nature, 386, 37

Hu, W., \& White, M. 1996a, Phys. Rev. Lett., 77, 1687 1996b, ApJ, 471, 30

Ivezić, Ž., et al. 2004, Astron. Nachr., 325, 583

Jaffe, A. H., et al. 2001, Phys. Rev. Lett., 86, 3475

Jain, B., \& Bertschinger, E. 1994, ApJ, 431, 495

Jungman, G., Kamionkowski, M., Kosowsky, A., \& Spergel, D. N. 1996, Phys.

Rev. Lett., 76, 1007

Kaiser, N. 1986, MNRAS, 219, 785 1987, MNRAS, 227, 1

Knop, R. A., et al. 2003, ApJ, 598, 102

Knox, L., Christensen, N., \& Skordis, C. 2001, ApJ, 563, L95 
Knox, L., \& Page, L. 2000, Phys. Rev. Lett., 85, 1366

Krauss, L. M., \& Chaboyer, B. 2003, Science, 299, 65

Landy, S. D., Shectman, S. A., Lin, H., Kirshner, R. P., Oemler, A. A., \& Tucker, D. 1996, ApJ, 456, L1

Landy, S. D., \& Szalay, A. S. 1993, ApJ, 412, 64

Lange, A. E., et al. 2001, Phys. Rev. D, 63, 042001

Lee, A. T., et al. 2001, ApJ, 561, L1

Linder, E. V. 2003, Phys. Rev. D, 68, 083504

Lupton, R., Gunn, J. E., Ivezić, Z., Knapp, G. R., Kent, S., \& Yasuda, N. 2001, in ASP Conf. Ser. 238, Astronomical Data Analysis Software and Systems X, ed. F. R. Harnden, Jr., F. A. Primini, \& H. E. Payne (San Francisco: ASP), 269

Ma, C., \& Fry, J. N. 2000, ApJ, 543, 503

Maor, I., Brustein, R., McMahon, J., \& Steinhardt, P. J. 2002, Phys. Rev. D, 65 , 123003

Matsubara, T. 2004, ApJ, 615, 573

Matsubara, T., \& Szalay, A. S. 2003, Phys. Rev. Lett., 90, 21302

McDonald, P., et al. 2004, ApJ, submitted (astro-ph/0407377)

Meiksin, A., White, M., \& Peacock, J. A. 1999, MNRAS, 304, 851

Miller, A. D., et al. 1999, ApJ, 524, L1

Miller, C., \& Batuski, D. J. 2001, ApJ, 551, 635

Miller, C., Nichol, R. C., \& Batuski, D. J. 2001, ApJ, 555, 68

Miller, C., Nichol, R. C., \& Chen, X. 2002, ApJ, 579, 483

Moodley, K., Bucher, M., Dunkley, J., Ferreira, P. G., \& Skordis, C. 2004 Phys. Rev. D, 70, 103520

Netterfield, C. B., et al. 2002, ApJ, 571, 604

Outram, P. J., Hoyle, F., Shanks, T., Croom, S. M., Boyle, B. J., Miller, L., Smith, R. J., \& Myers, A. D. 2003, MNRAS, 342, 483

Outram, P. J., Shanks, T., Boyle, B. J., Croom, S. M., Hoyle, F., Loaring, N. S., Miller, L., \& Smith, R. J. 2004, MNRAS, 348, 745

Padmanabhan, T. 2005, Curr. Sci., 88, 1057

Peacock, J. A., \& Smith, R. E. 2000, MNRAS, 318, 1144

Pearson, T. J., et al. 2003, ApJ, 591, 556

Peebles, P. J. E. 1980, The Large-Scale Structure of the Universe (Princeton: Princeton Univ. Press)

Peebles, P. J. E., \& Yu, J. T. 1970, ApJ, 162, 815

Peiris, H. V., et al. 2003, ApJS, 148, 213

Percival, W. J., et al. 2001, MNRAS, 327, 1297

2002, MNRAS, 337, 1068 2004, MNRAS, 353, 1201

Perlmutter, S., et al. 1999, ApJ, 517, 565

Pier, J. R., et al. 2003, AJ, 125, 1559

Riess, A. G., et al. 1998, AJ, 116, 1009 2004, ApJ, 607, 665
Roukema, B. F., Mamon, G. A., \& Bajtlik, S. 2002, A\&A, 382, 397 Rybicki, G. B., \& Press, W. H. 1994, preprint (comp-gas/9405004)

Scherrer, R. J., \& Weinberg, D. H. 1998, ApJ, 504, 607

Schlegel, D. J., Finkbeiner, D. P., \& Davis, M. 1998, ApJ, 500, 525

Schneider, D. P., et al. 2005, AJ, 130, 367

Scoccimarro, R. 2004, Phys. Rev. D, 70, 083007

Scoccimarro, R., \& Sheth, R. K. 2002, MNRAS, 329, 629

Scoccimarro, R., Sheth, R. K., Hui, L., \& Jain, B. 2001, ApJ, 546, 20

Seljak, U. 1996, ApJ, 463, 1 2000, MNRAS, 318, 203

Seljak, U., \& Zaldarriaga, M. 1996, ApJ, 469, 437

Seljak, U., et al. 2005, Phys. Rev. D, 71, 103515

Seo, H., \& Eisenstein, D. J. 2003, ApJ, 598, 720 . 2005, ApJ, 633, 575

Silk, J. 1968, ApJ, 151, 459

Smith, J. A., et al. 2002, AJ, 123, 2121

Smith, R. E., et al. 2003, MNRAS, 341, 1311

Spergel, D. N., et al. 2003, ApJS, 148, 175

Stompor, R., \& Efstathiou, G. 1999, MNRAS, 302, 735

Stoughton, C., et al. 2002, AJ, 123, 485

Strauss, M. A., et al. 2002, AJ, 124, 1810

Sunyaev, R. A., \& Zel'dovich, Ya. B. 1970, Ap\&SS, 7, 3

Sutherland, W., et al. 1999, MNRAS, 308, 289

Tegmark, M. 1997, Phys. Rev. Lett., 79, 3806

Tegmark, M., Hamilton, A., Strauss, M., Vogeley, M., \& Szalay, A. 1998, ApJ, 499, 555

Tegmark, M., Hamilton, A. J. S., \& Xu, Y. 2002, MNRAS, 335, 887

Tegmark, M., et al. 2004a, ApJ, 606, 702 . 2004b, Phys. Rev. D, 69, 103501

Tonry, J. L., et al. 2003, ApJ, 594, 1

Turner, M. S., \& White, M. 1997, Phys. Rev. D, 56, 4439

Virey, J. M., Taxil, P., Tilquin, A., Ealet, A., Fouchez, D., \& Tao, C. 2004, Phys. Rev. D, 70, 043514

Weller, J., \& Albrecht, A. 2002, Phys. Rev. D, 65, 103512

White, S. D. M., Navarro, J. F., Evrard, A. E., \& Frenk, C. S. 1993, Nature, 366, 429

Yahata, K., et al. 2005, PASJ, 57, 529

York, D. G., et al. 2000, AJ, 120, 1579

Zaldarriaga, M., \& Seljak, U. 2000, ApJS, 129, 431

Zaldarriaga, M., Seljak, U., \& Bertschinger, E. 1998, ApJ, 494, 491

Zehavi, I., et al. 2005a, ApJ, 621, 22 2005b, ApJ, 630, 1 